\title{
Tabularia
}

\section{À partir de la diffusion de trois poèmes hagiographiques, identification des centres carolingiens ayant influencé l'œuvre de Dudon de Saint-Quentin}

From the diffusion of three hagiographic poems, identification of Carolingian centres bearing an influence on the works of Dudo of Saint-Quentin

\section{Stéphane Lecouteux}

\section{OpenEdition \\ Journals}

Édition électronique

URL : http://journals.openedition.org/tabularia/1496

DOI : $10.4000 /$ tabularia. 1496

ISSN : $1630-7364$

Éditeur :

CRAHAM - Centre Michel de Boüard, Presses universitaires de Caen

\section{Référence électronique}

Stéphane Lecouteux, «À partir de la diffusion de trois poèmes hagiographiques, identification des centres carolingiens ayant influencé l'œuvre de Dudon de Saint-Quentin », Tabularia [En ligne], Écrire I'histoire au Moyen Âge, mis en ligne le 26 avril 2005, consulté le 19 avril 2019. URL : http:// journals.openedition.org/tabularia/1496; DOI : 10.4000/tabularia.1496 


\title{
À partir de la diffusion de trois poèmes hagiographiques, identification des centres carolingiens ayant influencé l'œuvre de Dudon de Saint-Quentin
}

\author{
From the diffusion of three hagiographic poems, \\ identification of Carolingian centres bearing \\ an influence on the works of Dudo of Saint-Quentin
}

\author{
Stéphane LECOUTEUX ${ }^{1}$ \\ 37, avenue du Grand Châtelet \\ 38100 Grenoble \\ lecouteux.stephmanue@wanadoo.fr
}

Résumé:

Afin d'identifier les centres culturels et intellectuels ayant influencé l'œuvre de Dudon de Saint-Quentin, il serait intéressant de mettre en lumière les lieux disposant de sources exploitées par cet auteur. Nous nous intéresserons ici exclusivement aux trois poèmes hagiographiques carolingiens identifiés par Leah Shopkow comme étant connus de Dudon: la Vita sancti Lamberti rédigée par l'évêque Étienne de Liège au début du $\mathrm{X}^{e}$ siècle, la Vita sancti Germani, écrite par le moine Heiric d'Auxerre dans la seconde moitié du IX ${ }^{e}$ siècle et le Carmen de sancto Cassiano, composé par un auteur anonyme sensiblement vers la mème époque. Nous verrons que le chanoine de Saint-Quentin, dont la collégiale d'appartenance était géographiquement proche des cathédrales de Cambrai et d'Arras, aurait pu connaître le texte d'Étienne par l'intermédiaire de l'une ou l'autre de ces deux églises. Mais Dudon aurait aussi bien pu effectuer un séjour à Liège, les voyages d'étude ne rebutant pas les clercs de son temps. Par ailleurs, la présence à Laon dès le $\mathrm{X}^{\mathrm{e}}$ siècle des deux dernières œuvres hagiographiques étudiées montre une fois encore les liens étroits que Dudon a entretenus avec cette cité. Le centre culturel et intellectuel traditionnel ayant le plus influencé le chanoine de Saint-Quentin semble donc bien être le milieu laonnois de l'an mil, un foyer fortement marqué à cette époque par la présence de deux prélats aux fortes personnalités: les évèques Roricon et Adalbéron. Ce constat permet d'entrevoir la possibilité d'une influence de deux ouvres contemporaines, composées à Laon dans la seconde moitié du $\mathrm{X}^{\mathrm{e}}$ siècle, sur l'historiographie de Dudon.

1. Je tiens à remercier Emmanuelle Doucet Lecouteux, Véronique Broust et le Comité de rédaction de la revue Tabularia pour la révision de cet article, ainsi que du précédent: leurs remarques, corrections et suggestions ont été nombreuses et judicieuses. Un grand merci également à l'ensemble du personnel de la Bibliothèque municipale de Grenoble pour sa disponibilité, et en particulier aux documentalistes et aux conservatrices des fonds anciens pour l'aide précieuse qu'elles m'ont apportée. 
Mots-clés: Dudon de Saint-Quentin, hagiographie, saint Lambert, Étienne de Liège, saint Germain, école d'Auxerre, Heiric d'Auxerre, saint Cassien, école de Laon, Roricon de Laon, Adalbéron de Laon.

Abstract:

In order to identify the traditional Carolingian centres bearing an influence on the works of Dudo de Saint-Quentin, it would be worthwhile to bring into relief the physical location of the sources used by the author. We shall concentrate here on the three Carolingian hagiographic poems identified by Leah Shopkow as being known to Dudo: Vita sancti Lamberti, written by bishop Stephen of Liège in the early tenth century, Vita sancti Germani, written by the monk Heiric of Auxerre in the second half of the ninth century, and Carmen de sancto Cassiano composed anonymously at around the same time. We shall see that the canon of Saint-Quentin, whose official collegiate church was geographically close to the Cambrai and Arras cathedrals, could have been familiar with Stephen's text through one or the other of those two churches. But Dudo could also very well have stayed for a while in Liège since the clerics of his time did not shun travelling in the course of their studies. Besides, the existence in Laon as early as the tenth century of the last two hagiographic works studied shows once more the close links binding Dudo to the city. The traditional centre of cultural and intellectual life which influenced most the canon of Saint-Quentin thus seems to be the intellectual circle at Laon in the year 1000, a circle on which two prelates with strong personalities very much left their marks - bishops Rorico and Adalbero. This suggests the possible influence of two contemporary works, composed in Laon in the second half of the tenth century, on the historiography of Dudo.

Keywords: Dudo of Saint-Quentin, hagiography, saint Lambert, Stephen of Liège, saint Germain, school of Auxerre, Heiric of Auxerre, saint Cassien, school of Laon, Rorico of Laon, Adalbero of Laon.

Le lieu d'étude et de formation de Dudon de Saint-Quentin reste aujourd'hui encore incertain. Les milieux intellectuels dans lesquels le chanoine a évolué demeurent presque aussi obscurs. Si certains liens unissant cet auteur à des villes telles que Saint-Quentin, Laon, Rouen et Fécamp ont pu être établis avec certitude, d'autres ont été suggérés sans pouvoir être réellement vérifiés : c'est en particulier le cas pour les cités de Liège et de Reims. Or, l'analyse des sources utilisées par le chanoine est peut-être une des clefs pour éclaircir ce «mystère »: nous savons aujourd'hui que Dudon puisa dans un éventail de textes extrêmement variés pour réaliser son travail, et certains étaient encore très peu connus de son vivant. En nous limitant volontairement aux trois œuvres hagiographiques mises en avant par Leah Shopkow ${ }^{2}$,

2. SHOPKOW, 1989, p. 20 col. 1. Dans son article, l'auteur montre de façon très convainquante l'influence d'un centre carolingien traditionnel sur Dudon : le chanoine utilise notamment trois poèmes hagiographiques, fruits de la renaissance carolingienne du IX siècle. Je remercie Martine Aubry, ingénieur d'études et responsable de la Bibliothèque du Centre de Recherche sur l'Histoire de l'Europe du Nord-Ouest (Université de Lille 3), pour la communication de ce très intéressant texte. Dans le cadre de notre étude, nous nous contenterons de suivre les affirmations de Leah Shopkow sans les remettre en question: seule une recherche linguistique approfondie permettrait de démontrer que Dudon s'est effectivement servi de ces trois poèmes; en effet, bien des termes et des thèmes peuvent être 
nous allons essayer de définir la zone géographique de prospection de Dudon: dans sa quête d'ouvrages destinés à lui fournir les matériaux pour la constitution de son De moribus et actis primorum Normanniae Ducum, le chanoine de Saint-Quentin ne s'est manifestement pas restreint à la consultation de recueils issus de la bibliothèque de sa collégiale. Les manuscrits renfermant au moins une de ces trois hagiographies semblent suffisamment peu nombreux pour permettre de définir leur zone géographique de diffusion au cours des $\mathrm{X}^{\mathrm{e}}$ et $\mathrm{XI}^{\mathrm{e}}$ siècles. Il conviendra de se restreindre, dans la mesure du possible, à l'époque contemporaine de Dudon. Nous nous intéresserons donc successivement à la Vita sancti Lamberti de l'évêque Étienne de Liège, à la Vita sancti Germani du moine Heiric d'Auxerre et enfin au Carmen de sancto Cassiano rédigé par un auteur anonyme. Ces études nous permettront de faire le point sur les hypothèses des formations de Dudon à Liège, à Reims ${ }^{3}$ et à Laon ${ }^{4}$.

\section{La Vita sancti Lamberti de l'évêque Étienne de Liège}

Selon Leah Shopkow, Dudon a été particulièrement influencé par l'œuvre d'Étienne de Liège tant au niveau de la structure et du style ${ }^{5}$ que du contenu ${ }^{6}$. Afin d'établir la zone géographique de diffusion de la Vita sancti Lamberti, nous allons tenter de retrouver, puis d'analyser, les différents manuscrits contenant cette œuvre, en précisant leur date et leur lieu de provenance ou d'appartenance. Devant l'étendue d'une telle démarche ${ }^{7}$, nous serons contraints de nous restreindre à l'ensemble des régions situées à l'ouest et au sud de Liège, ce qui devrait suffire puisque la présence de Dudon n'a été jusqu'ici attestée nulle part ailleurs.

parvenus auprès de Dudon par des intermédiaires, comme c'est le cas pour Virgile, qui tantôt a été sa source directe et tantôt sa source indirecte grâce notamment aux médiations de Prudence et de Fortunat (à ce sujet, voyez BouET, 1990, p. 215-236).

3. Les formations de Dudon à Liège, sous l'évêque Notger, et à Reims, sous l'écolâtre Gerbert d'Aurillac, ont été suggérées par plusieurs auteurs (cf. SHOPKOW, 1989, p. 21 col. 2 ; p. 25 col. 2 - 27 col. 2 ; Dictionnaire des biographies, 1993, p. 110). Pour les liens de Dudon avec Saint-Quentin, Rouen, Fécamp, Reims et Laon, voir BAUDUIN, 2004, p. 63-64.

4. Lecouteux, 2004, p. 36 (voir également p. 21 et p. 32-33).

5. Ces deux auteurs ont recours à la structure caractéristique du prosimetrum: des pièces de vers sont introduites au milieu du texte principal composé en prose rimée et rythmée (SHOPKOW, 1989, p. 26 col. 1 - 27 col. 1).

6. Le chanoine de Saint-Quentin s'inspire en effet d'événements de la Vita sancti Lamberti pour constituer son histoire des premiers ducs normands. D'après Leah Shopkow (1989, p. 27 col. 1 et col 2 et p. 25 col. 1), l'influence de l'œuvre d'Étienne sur Dudon transparait au niveau de la description du jeune Guillaume, de la narration de son assassinat et de ses funérailles, ainsi qu'au cours du discours d'adieu formulé par le duc Richard.

7. Au total, nous avons pu comptabiliser 36 manuscrits renfermant la Vita sancti Lamberti rédigée par Étienne de Liège. 


\section{Étienne et l'école de Liège ${ }^{8}$}

Les débuts de l'école cathédrale liégeoise semblent relativement modestes ${ }^{9}$. Certes, le passage de savants proches du palais impérial, tel que Sedulius Scottus au milieu du IX ${ }^{\mathrm{e}}$ siècle, a fait de la cité un centre intellectuel prestigieux dès cette époque ${ }^{10}$. Mais le séjour de l'Irlandais ne dure que quelques années, et aucune véritable école liégeoise ne paraît avoir survécu à son départ ${ }^{11}$. Les premiers signes de l'existence d'écoles apparaissent en fait au début du $\mathrm{X}^{\mathrm{e}}$ siècle, sous l'évêque Étienne, grâce aux échanges fructueux qui semblent naître entre le monastère de Lobbes et la cathédrale de Liège. Dès lors, et tout au long du $\mathrm{X}^{\mathrm{e}}$ siècle, des personnages tels que Rathier, Eracle, Folcuin, Hériger et Notger sont responsables d'échanges de plus en plus réguliers entre ces deux endroits: les écoles cathédrale et monastique se lient progressivement l'une à l'autre ${ }^{12}$. Mais c'est surtout sous les épiscopats d'Eracle (959-972) et de Notger (9721008) de Liège et sous les abbatiats de Folcuin (965-990) et d'Hériger (990-1007) de Lobbes que les écoles furent les plus prospères et les plus réputées ${ }^{13}$. Cette période correspond à celle où Liège devint une principauté ecclésiastique (en 985) et où Dudon fut formé.

8. Sur cet auteur, voir Histoire Littéraire de la France, 1867, t. VI, p. 168-172 ; Gallia christiana, 1876, t. III, p. 836-837. Sur la Vita sancti Lamberti, voir: MANITIUS, 1974, p. 705 ; ÉTIENNE DE LIÈGE, 1880, PL 132, p. 643-660; ÉTIENNE DE LIĖGE, MGH PLAC, 1899, t. 4, p. 232-233. Sur les liens de Liège et Lobbes avec la province de Reims, voyez SOT, 1993, p. 78-79.

9. RICHÉ, 1979, p. 165.

10. RICHÉ, 1979, p. 92 ; Dictionnaire du Moyen Âge, 2002, p. 1314; SHOPKOW, 1989, p. 27 col. 1. Le maitre irlandais Sedulius Scottus est présent à Liège sous les évêques Hartgar et Francon, probablement de 847 à 859 . À la fois poète, grammairien, théologien, exégète et moraliste, il connaît le grec et il est le chef de file d'une colonie de savants irlandais (Dermoth, Fergus, Blandus, Marcus et Bentchell). Sedulius entretient des relations avec les grands laïcs et ecclésiastiques de son temps, et il tient le rôle de poète officiel du milieu impérial : il côtoie à Liège l'impératrice Ermengarde, l'empereur Lothaire, Louis le Germanique et Charles le Chauve lors de leurs venues en ce lieu.

11. C'est vers 860 que Sedulius semble avoir quitté Liège pour rejoindre l'archevêque de Milan Tado (860-868). Suite à ce départ, on ne trouve guère de traces d'un enseignement dans les écoles liégeoises avant le début du $\mathrm{X}^{\mathrm{e}}$ siècle : la formation d'élèves devenant maîtres à leur tour n'a visiblement pas, ou peu, existé à Liège dans la seconde moitié du IX ${ }^{\mathrm{e}}$ siècle.

12. Rathier étudie à Lobbes, monastère avec lequel il reste en contact durant toute son existence. Il vécut une vie tumultueuse au cours de laquelle il fut notamment évêque de Vérone (931-934; 946-948; 961-968) et évêque de Liège (953-955) : Dictionnaire du Moyen Âge, 2002, p. 1177-1178; Histoire Littéraire de la France, 1867, t. VI, p. 339-383. Avant de devenir abbé de Lobbes, Hériger enseigne dans l'école de ce monastère sous l'abbé Folcuin (965-990), un ancien moine du monastère SithiuSaint-Bertin (Histoire Littéraire de la France, 1867, t. VI, p. 384-386 et 451-459; c'est par erreur que le moine Folcuin de Saint-Bertin et l'abbé Folcuin de Lobbes sont perçus comme deux individus distincts dans cette œuvre).

13. RICHÉ, 1979, p. 165-166; Histoire Littéraire de la France, 1867, t. VII, p. 29, 194-208 et 208-216; SHOPKOW, 1989, p. 26 col. 1. Les évêques Eracle et Notger furent particulièrement sensibles à la formation des enfants, tant à Liège (dans les écoles de la cathédrale et de la collégiale Saint-Jean) qu'à Lobbes (relation étroite avec l'écolâtre puis abbé Hériger, qui est à la fois théologien, poète, hagiographe et mathématicien). 
Mais revenons à Étienne de Liège, né peu avant 850. Apparenté à la dynastie carolingienne et à la haute aristocratie franque ${ }^{14}$, il est formé dès 864 à l'école palatine du roi Charles le Chauve $(+877)$, devenu empereur en 875 . C'est à cette cour qu'il étudie tout d'abord auprès du savant Mannon ${ }^{15}$, puis auprès de Robert, évêque de Metz (883-917) ${ }^{16}$. Ce dernier était un ancien condisciple - et peut-être même élève de Notker le Bègue (v. 840-912) à l'école du prestigieux monastère de Saint-Gall, situé sur les bords du lac de Constance ${ }^{17}$. Notker est un moine réputé pour ses qualités de versificateur et de poète : il a rédigé une Vita sancti Galli en prose et en vers (prosimetrum). Il a également effectué des travaux musicaux, séquences et tropes, dans son Liber Hymnorum ${ }^{18}$. Ces disciplines et cette culture, acquises par Robert de Metz, ont été transmises à leur tour à Étienne, qui put se constituer une solide formation poétique, musicale et liturgique: on retrouve dans ses œuvres un certain nombre de similitudes avec celles issues du milieu messin et du monastère de SaintGall aux IX ${ }^{e}$ et $X^{e}$ siècles. C'est également sous Robert qu'il devient chanoine de la cathédrale de Metz vers 888. Grâce à ses origines, les rois carolingiens de Francie occidentale et de Francie orientale lui confient peu à peu la charge de plusieurs abbayes, notamment Saint-Mihiel, Saint-Evre de Toul et Moustier-sur-Sambre. Tout en gardant la direction de ces différents monastères, il apporte la tradition musicale messine à Liège où il devient évêque vers 901, à la mort de Francon. Louis l'Enfant (893-911), dernier roi carolingien de Germanie, lui confirme en 908 la possession des abbayes de Lobbes et de Fosses, ainsi que toutes les donations faites à son église par les rois et les empereurs précédents ${ }^{19}$. Après 911 , lorsque le diocèse de Liège est momentanément

14. Étienne était un proche parent des comtes Gérard et Matfried, mais également du roi Charles le Simple (879-929). Il apparaît dans un diplôme de ce roi en ces termes : Stephani venerabilis Tungrorum Episcopi, nostrae consanguinitatis affinis dilectissimi. Une prétendue parenté avec Gérard de Brogne, célèbre réformateur de plusieurs abbayes, a été inventée pour accroître sa renommée, ou par confusion avec le premier des deux comtes précédemment cités.

15. Mannon, qui semble avoir succédé à Jean Scot, dit Erigène, à la tête de l'école palatine, enseigna les lettres et la philosophie à Étienne. Il fut également écolâtre à Laon $(\sim 875-900)$, où il fut ordonné prêtre en 876 (SOT, 1993, p. 59). On ne sait si Étienne a suivi l'enseignement de Mannon à Metz ou à Laon.

16. Histoire Littéraire de la France, 1867, t. VI, p. 75, 156-157 et 172. Il semble que Robert de Metz puisse être l'auteur d'une Vie de saint Théodore, évêque de Sion (BHL 8088 et 8088a, fêté le 16 ou 26 août). Étienne dédia à cet évêque et maître son Liber capitularis, sorte de recueil de textes liturgiques (bréviaire/antiphonaire), constitué de leçons avec répons, versets, capitules, oraisons, collectes, hymnes, etc., où l'auteur précise les pièces destinées à être chantées. Robert est incontestablement le maître qui a le plus influencé l'œuvre d'Étienne.

17. SOT, 1993, p. 78.

18. Dictionnaire du Moyen Âge, 2002, p. 1004; Dictionnaire des biographies, 1993, p. 233-234; Dictionnaire des lettres françaises, 1964, p. 552-553; Histoire Littéraire de la France, 1867, t. VI, p. 75, 134-144; CHARTIER, Yves, «Naissance de la séquence au IX"e siècle : Notker de Saint-Gall ».

19. Gallia Christiana, 1874, t. XIII, col. 1273 et suiv; col. 1074 et t. III, col. 836-837. Les possessions de l'église de Liège, au milieu du $\mathrm{X}^{\mathrm{e}}$ siècle, se situent essentiellement le long de la Meuse (Arches aujourd'hui Charleville - Theux et sa forêt) et de la Sambre (domaines de Pont-de-Loup et de Marchiennes-au-Pont). L'église possède des droits et des biens situés principalement dans les grandes localités du pays mosan (Liège, Tongres, Maastricht, Huy, Namur et Dinant), ainsi que les abbayes 
rattaché à la Francie occidentale ${ }^{20}$, Charles le Simple accorde à Étienne l'abbaye d'Hastière et lui confirme celle de Saint-Rombaud de Malines. L'évêque de liège assure ainsi un lien intellectuel et culturel entre la partie occidentale et la partie orientale de l'ancien Empire carolingien, divisé depuis le partage de Verdun en 843 et dont la Lotharingie était devenue l'enjeu. Étienne est surtout célèbre en tant qu'hagiographe et liturgiste puisque ses œuvres portent essentiellement sur ces deux domaines ${ }^{21}$; il compose plusieurs offices religieux ${ }^{22}$, et il joue un rôle important dans le développement de la musique liturgique ${ }^{23}$. Il occupe le siège épiscopal de Liège pendant près de 20 ans, et meurt en $920^{24}$.

Parmi l'ensemble de la production hagiographique relative à saint Lambert, l'œuvre d'Étienne de Liège a connu une grande renommée: elle fut privilégiée par les clercs et les chanoines de la cité épiscopale à partir du début du $\mathrm{X}^{\mathrm{e}}$ siècle et au-delà du XII ${ }^{e}$ siècle. Une telle longévité, malgré plusieurs tentatives effectuées pour remplacer ce texte par un autre, est réellement remarquable. Est-ce son côté "politiquement correct ", n'accablant pas les Carolingiens et l'Empire, qui a fait son succès? Est-ce le style littéraire choisi, très travaillé, qui a su séduire son auditoire? L'emploi du prosimetrum était surtout remarquablement bien adapté pour l'office liturgique de la fête du saint patron liégeois : les poèmes et les pièces de vers, parfois empruntés littéralement au Carmen de sancto Landberto ${ }^{25}$, interrompent de façon régulière le texte principal rédigé en prose rimée et rythmée, ce qui le rend plus vivant lors de la liturgie communautaire. Étienne l'avait conçu pour qu'il s'intègre complètement à l'Office qu'il avait composé sur saint Lambert, en alternant les parties lues et chantées, et c'est vraisemblablement l'harmonie de cet ensemble qui a permis la longévité de son œuvre. Toutefois, la parenté d'Étienne avec les Carolingiens n'est probablement pas étrangère à la diffusion de sa Vita sancti Lamberti au sein de la noblesse liée à cette

de Saint-Hubert, de Lobbes, de Fosses et d'Aldeneik (Histoire de la principauté de Liège, 2002, p. 14). Pour localiser ces lieux, voyez la carte 1 .

20. Le rattachement de la Lotharingie au royaume de Charles le Simple sera effectif de 911 à 925 (FLODOARD, 1906, an. 925, p. 32).

21. RICHÉ, 1979, p. 165.

22. AUDA, 1923, p. 1-211 : Étienne de Liège dédie son Office de l'Invention de saint Étienne (p. 42-66) à son maitre l'évêque Robert de Metz (le premier martyr de la chrétienté était particulièrement vénéré à Metz: la cathédrale avait été consacrée sous l'invocation de ce saint); il compose également un Office de la sainte Trinité, tant pour les Vêpres, que les Matines et les Laudes de cette fête (p. 67-121). Enfin, Étienne réalise un Office de saint Lambert, patron de sa propre cathédrale (p. 122-197).

23. Étienne renouvelle le chant de l'office religieux et compose les premiers documents musicaux connus du diocèse de Liège, fruits de sa formation à Metz (dès 800 apparaît le Tonaire de Metz donnant le premier exemple d'une classification du chant liturgique en 8 modes). Avec Hucbald de Saint-Amand, il est considéré comme le principal instigateur de la musique liturgique au début du $\mathrm{X}^{\mathrm{e}}$ siècle. Les deux hommes entretenaient d'ailleurs des relations littéraires suivies, et Hucbald a vraisemblablement connu la Vita sancti Lamberti composée par Étienne.

24. Étienne est mort le 19 mai 920 et fut enterré dans la crypte de la cathédrale Saint-Lambert de Liège.

25. SHоркоW, 1989, p. 27 col. 1. Étienne a remplacé certains passages de l'ancienne Vita par des vers tirés du Carmen de sancto Landberto - œuvre dont il fut l'instigateur - qu'il a intercalés dans sa prose (Histoire Littéraire de la France, 1867, t. VI, p. 170). 
dynastie. Ainsi, l'œuvre hagiographique de l'évêque de Liège avait de bonnes raisons d'être diffusée en dehors de son diocèse. D'autant qu'Étienne, abbé de plusieurs monastères et chargé de restaurer diverses abbayes de part et d'autre de la frontière séparant les royaumes issus de l'Empire carolingien, entretenait des contacts avec des personnages influents en ces différents endroits. Si plusieurs manuscrits du diocèse de Liège nous sont parvenus, un certain nombre de manuscrits lui étant extérieurs ont également survécu : il convient à présent de les étudier, en nous concentrant surtout sur les plus anciens, présents à l'ouest et au sud de Liège.

\section{Les manuscrits de la Vie de Saint Lambert (Vita sancti Lamberti) d'Étienne de Liège}

Ce sont les trois manuscrits les plus anciens, datant du $\mathrm{X}^{\mathrm{e}}$ siècle, qui retiendront en premier lieu notre attention : c'est sans doute par leur intermédiaire que la diffusion initiale de cette Vita fut possible.

Le contenu du premier manuscrit trahit une origine liégeoise ${ }^{26}$. En plus de la vie de saint Lambert, évêque de Tongres, rédigée par Étienne (BHL 4683), il contient deux autres textes sur ce saint: le Carmen, composé à la demande d'Étienne (BHL 4682), et un récit de miracles (BHL 4689), vraisemblablement composé vers la même époque. Il renferme également plusieurs autres vies de saints de ce diocèse, dont saint Remacle, évêque de Tongres et abbé de Stavelot-Malmédy (BHL 7113 et 7120, fêté le 3 septembre), saint Amand, évêque de Tongres (BHL 0332, fêté le 6 février), saint Hubert, évêque de Liège (BHL 3994, 3995 et 3996, fêté le 3 novembre) et saint Trond, qui donna son nom au monastère et à la ville situés à l'ouest de Tongres (BHL 8321, fêté le 23 novembre) ${ }^{27}$. En revanche, il ne contient pas la version de la Vie de saint Remacle rédigée par Hériger de Lobbes, ni l'une des nombreuses vies de saints composées dans la mouvance de Liège durant la seconde moitié du $\mathrm{X}^{\mathrm{e}}$ siècle, notamment sous les évêques Rathier, Eracle et $\operatorname{Notger}^{28}$.

Le deuxième manuscrit du $\mathrm{X}^{\mathrm{e}}$ siècle est un petit recueil qui appartenait à l'abbaye de Saint-Mihiel ${ }^{29}$. La Vita est précédée de 24 sermons et débute par un prologue composé par l'évêque Étienne de Liège. Un second texte hagiographique fut ajouté à la fin par la suite (Passio sancti Mauritii, BHL 5744, fêté le 22 septembre) : il ne

26. Le ms Bruxelles KBR 14650-14659 (3236), constitué au X $\mathrm{X}^{\mathrm{e}}$ siècle, est vraisemblablement originaire de la cathédrale de Liège : certaines des vies le constituant sont rares et n'apparaissent que dans des manuscrits proches du milieu liégeois. C'est le cas notamment du Carmen de sancto Lamberto (BHL 4682), des miracles relatifs à ce saint (BHL 4689) et de la Vie de saint Trond (BHL 8121). On ne sait précisément quand l'évêché de Tongres/Maastricht fut transféré à Liège.

27. Ce manuscrit contient également la Vita Leodegarii Augustodunensis episcopi d'Ursinus (BHL 4851, fêté le 2 octobre) : cette vie, portant sur un évêque d'Autun, est la seule qui ne soit pas en rapport direct avec le milieu liégeois.

28. L'âge et le contenu de ce manuscrit laissent supposer qu'il pourrait s'agir d'un recueil constitué en vue de réaliser les Gestes des évêques de Liège à la fin du $\mathrm{X}^{\mathrm{e}}$ siècle; du fait de son ancienneté, on peut supposer qu'il n'a pas ou peu existé d'intermédiaires entre ce ms et l'autographe d'Étienne.

29. Il s'agit du ms Saint-Mihiel BP 20 part. 2. Cf. Catalogue général des manuscrits des bibliothèques..., 1861, t. III (Saint-Mihiel), p. 518-519. 
faisait donc pas partie du contenu initial du recueil, et par conséquent n'offre que peu d'intérêt pour notre étude.

Nous avons vu que l'abbaye de Saint-Mihiel ${ }^{30}$, située en Lotharingie, au sud de Verdun, a eu Étienne comme abbé vers 895 avant qu'il n'accède au siège épiscopal de Liège environ six ans plus tard. Il est probable que l'évêque de Liège a transmis son œuvre hagiographique à son monastère, auquel il resta attaché jusqu'à sa mort: les 24 sermons qui précédent la Vita sont peut-être également l'œuvre de ce grand liturgiste ${ }^{31}$.

Bien que nous n'en ayons pas la trace, il est fort probable que les autres abbayes dirigées ou restaurées par Étienne ont également disposé de sa Vita de son vivant. Si seul un manuscrit de Saint-Mihiel nous est parvenu, on remarque que l'auteur de la légende de saint Lambert dans les Annales de Lobbes disposait de cette œuvre pour constituer son texte ${ }^{32}$. De même, Sigebert l'utilisa lui aussi dans son monastère de Gembloux, qui dépendait alors de Liège, comme source pour constituer sa version de la Vie de saint Lambert un siècle plus tard ${ }^{33}$. Ainsi, dès les $\mathrm{X}^{\mathrm{e}}$ et $\mathrm{XI} \mathrm{I}^{\mathrm{e}}$ siècles, plusieurs abbayes liées à Étienne ou à l'évêché de Liège disposaient de manuscrits renfermant la Vita sancti Lamberti identifiée par les Bollandistes sous la référence BHL 4683.

Le troisième manuscrit du $\mathrm{X}^{\mathrm{e}}$ siècle est un petit recueil appartenant à l'abbaye Saint-Vaast d'Arras ${ }^{34}$. Outre la Vie de saint Lambert, évêque de Liège, il contient également la Vie de saint Remi, archevêque de Reims, rédigée par son successeur Hincmar (BHL 7153, 7154, 7155, 7156, 7157, 7158 et 7159, fêté le 1 octobre), une Vie de saint Nicaise, archevêque de Reims (BHL 6078, fêté le 14 décembre) et une Vie de saint Maur, rédigée par Odon de Glanfeuil (BHL 5773, fêté le 15 janvier) ${ }^{35}$. Or, on retrouve

30. Au X $\mathrm{X}^{\mathrm{e}}$ siècle, Saint-Mihiel était rattachée à l'abbaye parisienne de Saint-Denis, et elle se trouvait ainsi indirectement entre les mains des princes Robertiens de France (futurs Capétiens) en tant que possession ancienne de cette abbaye (cf. L'abbaye de Gorze au X'e siècle, 1993, p. 12 et 80 ; Gallia Christiana, 1874, XIII, col. 1273 et suiv.). Serait-ce du fait des liens de Saint-Mihiel avec Saint-Denis que l'œuvre d'Étienne de Liège s'est ensuite répandue dans certaines abbayes parisiennes (notamment Saint-Germain-des-Prés et Saint-Victor-de-Paris) ? Voici la liste des manuscrits " parisiens » renfermant l'œuvre d'Étienne: mss Paris BnF fonds lat. 5308, 5278, 11759 et 14651 ; mss Paris BnF fonds fr. 17229 et 23117 ; mss Paris BnF anciens fonds fr. 411, 988 et 413 . Tous sont relativement tardifs (ils datent du XII ${ }^{\mathrm{e}}$ au XV ${ }^{\mathrm{e}}$ siècle) et ne sont sans doute pas d'origine "parisienne».

31. Ce recueil contiendrait donc la plus ancienne Vie de Saint Lambert rédigée par Étienne qui nous soit parvenue; cependant, il s'agissait peut-être initialement d'un sermonnaire, dans lequel la Vita aurait été ajoutée peu après.

32. Ce texte semble avoir été composé sous l'épiscopat de Notger de Liège (972-1008), aux alentours de l'année 982 (KUPPER, 1984, p. 5-49). À cette époque, Folcuin, était abbé de Lobbes (965-990), et Hériger enseignait dans l'école de ce même monastère : l'un de ces deux écrivains prolifiques fut peut-être l'auteur de ce texte ou, du moins, encouragea sa composition.

33. Ibidem. La Vita de Sigebert de Gembloux a été référencée sous deux numéros par les Bollandistes (BHL 4686 et 4687). Au total, 13 manuscrits provenant essentiellement de Belgique ont été identifiés ( 2 sont du XII ${ }^{e}$ siècle et 3 du XIII ${ }^{e}$ siècle).

34. Ms Arras BP 199 (189), Xe siècle. Cf. Catalogue général des manuscrits des bibliothèques..., 1872, t. IV (Arras), p. 89-90.

35. Ce manuscrit a énormément souffert: il ne reste malheureusement aujourd'hui que quelques feuillets des vies de saint Nicaise et de saint Lambert, ce qui risque de rendre tout travail paléographique et de comparaison de manuscrits extrêmement difficile. 
la plupart de ces textes dans un manuscrit du $\mathrm{XI}^{\mathrm{e}}$ siècle ayant appartenu à l'abbaye de Marchiennes ${ }^{36}$ : une partie de la Vie de saint Remi d'Hincmar (uniquement les BHL 7155, 7156 et 7157), la Vie de saint Lambert d'Étienne de Liège et la Vie de saint Maur. Si la Vie de saint Nicaise n'apparaît pas dans ce manuscrit, en revanche, on trouve la Vie de saint Germain d'Auxerre de Constance de Lyon (BHL 3454, fêté le 31 juillet), une Vie de saint Mamert, abbé d'Auxerre (BHL 5201, fêté le 20 mars ou le 20 avril), une Vie de Benoît de Nursie, abbé du Mont-Cassin (BHL 1117, fêté le 21 mars ou 11 juillet) et une Vie du prêtre Jérôme rédigée par un moine du MontCassin (BHL 3870, fêté le 30 septembre). Il serait intéressant de déterminer si le manuscrit de Marchiennes a été copié sur celui de Saint-Vaast d'Arras (avec ou sans intermédiaires) ou si ces deux manuscrits ont en fait un ancêtre commun. En effet, leur contenu - qui regroupe des saints originaires de Reims, Liège et Auxerre, ainsi que des saints chers aux moines bénédictins - et leur localisation dans la vallée de la Scarpe font aussitôt penser à Hucbald de Saint-Amand: il n'est pas impossible que ce moine, lié à ces différents endroits, ait pu constituer un recueil regroupant ces vies de saints pour ses travaux hagiographiques réalisés autour de l'an $900^{37}$; des copies et des extraits plus ou moins complets de ce recueil auraient ensuite pu circuler dans les différentes communautés monastiques bénédictines de la vallée de la Scarpe (Saint-Amand, Marchiennes, Anchin, Hasnon, Saint-Vaast d'Arras) à partir de cette époque ${ }^{38}$. En effet, plusieurs manuscrits plus récents issus de ces abbayes et renfermant la Vita sancti Lamberti d'Étienne nous sont parvenus: ils proviennent de Saint-Vaast d'Arras, d'Anchin et même de l'abbaye augustinienne du Mont-SaintEloi, elle aussi située sur la Scarpe, au nord-ouest d'Arras ${ }^{39}$. Le regroupement de ces manuscrits, datant du $\mathrm{X}^{\mathrm{e}}$ au XIV $\mathrm{X}^{\mathrm{e}}$ siècle, sur toute l'étendue de cette vallée, dans les diocèses d'Arras et de Cambrai, est remarquable: une telle concentration ne se retrouve qu'autour de Liège, sur une étendue cependant plus réduite.

36. Ms Douai BP 856, XI siècle. Cf. Catalogue général des manuscrits des bibliothèques..., 1878, t. VI (Douai), p. 607-608 : bien que l'écriture soit d'un style du XIe siècle (datation adoptée par les Bollandistes), ce catalogue date le manuscrit du XII ${ }^{\mathrm{e}}$ siècle. Ce même catalogue attribue d'ailleurs par erreur cette Vita au chanoine Nicolas (voir commentaires relatifs aux folios 19v à 26), ce qui explique certainement cette datation tardive erronée.

37. J'envisage de publier prochainement un article abordant cette question.

38. Je remercie Jean-Pierre GERZAGUET pour les précisions intéressantes qu'il a pu m'apporter au sujet des diocèses d'Arras et de Cambrai aux IX ${ }^{\mathrm{e}}, \mathrm{X}^{\mathrm{e}}$ et $\mathrm{XI}^{\mathrm{e}}$ siècles, ainsi que pour la communication d'informations au sujet des différentes communautés bénédictines de la vallée de la Scarpe, sur lesquelles portait son habilitation à diriger des recherches. Voir Gallia christiana, 1876, t. III, p. 119 (Cambrai), 374 (Arras), 395 (Marchiennes), 399 (Hasnon) et 408 (Anchin).

39. Le ms Douai BP 864, appartenant à l'abbaye d'Anchin et daté du XIII' siècle contient la Vita sancti Lamberti d’Étienne aux folios 33v-42v: cf. Catalogue général des manuscrits des bibliothèques..., 1878, t. VI (Douai), p. 617-618. Les mss Arras BP 14 (23) et 573 (462), appartenant respectivement aux abbayes du Mont-Saint-Eloi et de Saint-Vaast-d'Arras, datent tous deux du XIII ${ }^{\text {e }}$ siècle et renferment ce texte respectivement aux folios 67-68 et 34-35v. On notera également la présence de cette œuvre dans la cathédrale d'Arras: le ms Arras BP 344 (961), datant du XIV siècle, provient de ce chapitre et contient la Vita aux folios 137-139. Cf. Catalogue général des manuscrits des bibliothèques..., 1872 t. IV (Arras), p. 20-21, 181-182 et 89-90. 
Un peu plus récent et originaire d'une région proche, le manuscrit de la Bibliothèque municipale de Cambrai 863-864 (ancien 767) apporte à lui seul des informations capitales pour notre étude ${ }^{40}$. Il s'agit d'un recueil de vies de saints en deux volumes, composé sous l'épiscopat de Gérard II de Cambrai (1076-1092) par le scriptorium de l'abbaye du Saint-Sépulcre ${ }^{41}$. Cette abbaye, fondée en 1064 par l'évêque Liebert de Cambrai (1049/51-1076) ${ }^{42}$, était à son origine en étroite relation avec sa cathédrale: les liens entre la bibliothèque du chapitre et le scriptorium du monastère, dont l'activité fut importante dans le dernier quart du $\mathrm{XI}^{\mathrm{e}}$ siècle, apparaissent au travers de plusieurs manuscrits nous étant parvenus ${ }^{43}$. Ce recueil n'échappe pas à cette règle, et divers manuscrits appartenant à la cathédrale de Cambrai aux $\mathrm{X}^{\mathrm{e}}$ et $\mathrm{XI}^{\mathrm{e}}$ siècles ont visiblement été mis à contribution par les moines du Saint-Sépulcre pour sa réalisation. Les textes hagiographiques qu'il renferme portent d'ailleurs essentiellement sur des saints figurant dans un martyrologe du chapitre de Cambrai, qui lui est presque contemporain ${ }^{44}$. Différents scribes sont intervenus à plusieurs reprises sur l'ensemble du manuscrit - parfois simplement sur quelques lignes - et on retrouve en particulier la main de Fulbertus peccator, le plus connu d'entre eux, en de nombreux endroits ${ }^{45}$. La répartition du travail entre plusieurs copistes n'empêcha en rien la réalisation de deux volumes homogènes, notamment au niveau de la décoration (initiales fourrées et lettres ornées); la table originelle, au début du ms 863 (folio 1),

40. Je souhaite remercier tout particulièrement Annie Fournier, du service des manuscrits et du livre ancien de la médiathèque municipale de Cambrai, pour la communication d'informations très précises sur les manuscrits 863-864 et 1224 de Cambrai (origine, appartenance et datation), ainsi que pour la transmission d'une copie du calendrier du martyrologe contenu dans le ms Cambrai B. 193 (188) et d'extraits de l'ouvrage de l'abbé Hélin.

41. Cf. Catalogue général des manuscrits des bibliothèques..., 1891, t. XVII (Cambrai), p. 343-348 (la Vita sancti Lamberti d'Étienne apparaît aux folios 161-167); Manuscrits datés des bibliothèques de France, 2000, t. I (Cambrai), p. 102-103. Remarque: les manuscrits de la Bibliothèque de Cambrai n’ont malheureusement pas été inventoriés par les Bollandistes.

42. Avant de devenir évêque, saint Liebert fut formé dans l'école cathédrale de Cambrai, réputée tout au long du XIe siècle, et notamment sous son oncle Gérard I (1013-1048/51), à qui il succéda. Liebert, considéré comme le plus illustre élève issu de l'école de Cambrai, semble avoir occupé la tête de celleci par la suite (Histoire Littéraire de la France, 1867, t. VII, p. 94 ; Gallia christiana, 1876, t. III, p. 14 22 et 118-119; Manuscrits datés des bibliothèques de France, 2000, t. I, p. XXII).

43. Sur l'abbaye du Saint-Sépulcre de Cambrai et son scriptorium, voyez les Manuscrits datés des bibliothèques de France, 2000, t. I (Cambrai), p. XXII-XXIII.

44. Le manuscrit Cambrai B. 193 (188) contient un martyrologe du milieu du XII ${ }^{\mathrm{e}}$ siècle (daté entre 1131 et 1173) à l'usage du chapitre de la cathédrale de Cambrai (calendrier aux folios 1 à 7). Cf. Manuscrits datés des bibliothèques de France, 2000, t. I (Cambrai), p. 42.

45. Ibidem. Fulbertus peccator est considéré comme «le» scribe de Saint-Sépulcre: il a souscrit explicitement l'intégralité des mss 215, 217, 218, 247 et 819 de la bibliothèque municipale de Cambrai; il a souscrit partiellement les mss 216 et 219 ; il a composé entièrement de façon anonyme le ms 504 ; enfin, il est intervenu partiellement et de façon anonyme dans les mss 807, 846, 863 et 864. Concernant ces deux derniers manuscrits, qui nous intéressent tout particulièrement ici, la main de Fulbert apparaît sur les folios $2 \mathrm{v}-7 \mathrm{v}, 41 \mathrm{v}, 200-254$ du ms 863 et sur les folios 1-5, 23v-25v, 124v-125v, 176v-208, 215-241 du ms 864. On notera que le ms 846, composé en partie par Fulbert, doit être considéré comme un complément au recueil 863-864, dont il est contemporain. 
confirme d'ailleurs qu'ils formaient dès leur origine un recueil unique ${ }^{46}$, comportant près de 150 vies de saints ${ }^{47}$.

Or, il existe un manuscrit, datant du milieu du XII ${ }^{\mathrm{e}}$ siècle, qui comporte un grand nombre de similitudes avec le recueil précédent sans dériver de celui-ci. Il s'agit du manuscrit 1224 de la Bibliothèque municipale de Cambrai (anciens 1097 et 1209), un lectionnaire copié à l'usage de l'un des chapitres de Cambrai: Saint-Géry ou le chapitre cathédral. De tout temps, ces deux établissements étaient étroitement liés, et leurs liturgies respectives étaient pratiquement identiques ${ }^{48}$. Ce lectionnaire, mélangeant à la fois des vies de saints apôtres, de martyrs et de confesseurs, respecte l'ordre du calendrier des fêtes de saints et il est resté en usage pendant de nombreux siècles ${ }^{49}$. Malheureusement mutilé, il n'en reste que deux fragments toutefois très précieux pour notre étude ${ }^{50}$.

Sur les 38 vies de saints subsistantes, 37 concernent des saints inscrits dans le martyrologe du XII e siècle de la cathédrale de Cambrai. De plus, on retrouve également 29 d'entre elles dans le recueil précédent du Saint-Sépulcre de Cambrai: 25 dans le premier volume (ms 863) et 4 (seulement) dans le second (ms 864). En poussant l'analyse plus loin, on a pu en déduire que le ms 1224 avait été constitué presque exclusivement à partir de trois manuscrits, qui ont malheureusement aujourd'hui disparu, et qui avaient également servi de base à la confection du ms $863^{51}$. Il est aujourd'hui possible de reconstituer presque intégralement ces trois manuscrits grâce aux mss 863 et 1224 de Cambrai, leurs deux descendants. Il s'agissait manifestement de deux lectionnaires et d'un recueil de vies de saints appartenant au chapitre

46. Les interventions de diverses mains ont eu lieu de façon indépendante des textes et de la structure matérielle: un scribe peut s'arrêter au milieu d'un texte, et une autre main prendre sa suite. Malgré les nombreux changements de main et de mise en page, ces deux volumes forment un recueil homogène de près de 600 feuillets $(292+273)$.

47. Nous procédons actuellement à une étude de ce recueil en vue d'une publication.

48. De nombreux manuscrits ayant appartenu à l'origine au chapitre de saint Géry sont parvenus par la suite dans les fonds de la cathédrale de Cambrai ; les liturgies de ces deux établissements se distinguant à peine, il est parfois malaisé de pouvoir attribuer avec certitude les manuscrits issus de l'un ou de l'autre lieu. Cf. Manuscrits datés des bibliothèques de France, 2000, t. I (Cambrai), p. 128-129; Catalogue général des manuscrits des départements..., 1891, t. XVII (Cambrai), p. 463465.

49. De nombreuses annotations indiquent que ce manuscrit fut en possession de la cathédrale de Cambrai, où il fut utilisé au moins jusqu'au XVII ${ }^{\mathrm{e}}$ siècle.

50. Comme pour le recueil 863-864, une étude sur ce manuscrit est également en cours, en vue d'une publication prochaine.

51. Il est probable que le manuscrit 1224 a été constitué afin de remplacer ces 3 manuscrits, devenus archaïques, pour obtenir un seul grand recueil regroupant dans l'ordre chronologique l'ensemble des vies de saints apôtres, martyrs et confesseurs que ces derniers contenaient de façon séparée. À quelques rares exceptions, tous ces saints figurent dans le martyrologe de la cathédrale de Cambrai constitué au XII ${ }^{\mathrm{e}}$ siècle. En fait, que ce soit lors de la constitution du recueil du Saint-Sépulcre dans le dernier quart du XI ${ }^{\mathrm{e}}$ siècle ou lors de la confection du lectionnaire pour un chapitre de Cambrai au milieu du XII e siècle, la démarche a été semble-t-il la même, puisqu'on s'est servi en priorité des 3 mêmes manuscrits comme source: ces lectionnaires et ces recueils étaient vraisemblablement très utilisées par le chapitre de la cathédrale sur cette période. 
cathédral de Cambrai ${ }^{52}$. Le premier lectionnaire, relatif aux apôtres, et le second, contenant des vies de saints martyrs et confesseurs, avaient tous deux la particularité de respecter l'ordre chronologique du sanctoral appliqué à Cambrai.

Dans le cadre de notre étude, c'est avant tout la reconstitution du second lectionnaire qui importe, puisque celui-ci contenait la Vita sancti Lamberti d'Étienne de Liège $^{53}$. En fait, c'est surtout la datation de ce manuscrit qui est primordiale: elle permettrait de définir l'époque à laquelle cette Vita était présente au plus tard à Cambrai. Nous savons, avec le ms Arras BP 199 (189) précédemment étudié, que l'œuvre d'Étienne était déjà connue à Arras dans le dernier quart du $\mathrm{X}^{\mathrm{e}}$ siècle. En était-il de même à Cambrai? À cette époque, et jusqu'à la fin du XI ${ }^{e}$ siècle, les diocèses de Cambrai et d'Arras étaient en effet réunis sous la direction du même évêque, avant d'être séparés peu après le décès de Gérard II (1076-1092) ${ }^{54}$. Or, l'ordre devant être observé par ces deux églises pour la célébration de l'office dominical et de l'office des fêtes de saints avait été établi de façon précise au moins dès la fin du $\mathrm{X}^{\mathrm{e}}$ siècle ou au commencement du suivant ${ }^{55}$. La mise en place de cet ordre explique vraisemblablement la constitution des lectionnaires dont nous venons de parler, puisque ces derniers suivaient précisément le calendrier des offices des saints : l'office dominical avait sans doute recours au lectionnaire relatif aux apôtres, considérés comme les saints les plus importants; le lectionnaire contenant des vies de saints martyrs et confesseurs correspondait indiscutablement à l'office des fêtes de saints suivant le sanctoral appliqué à Cambrai.

Autour de l'an mil, les évêques de Cambrai étaient Rothard (976-995) et Herluin (995-1012). Or, tous deux avaient été formés par Notger de Liège (972-1008), qui joua vraisemblablement un rôle actif pour leur établissement sur ce siège épiscopal $^{56}$. Les liens de Cambrai avec Liège, notamment via Lobbes, étaient également très importants à cette époque ; Rothard et Herluin, élèves de Notger, conservèrent une relation étroite avec leur maître, et il est probable qu'ils ont cherché à mettre en

52. Nous nous étendrons davantage sur ce sujet dans le cadre de l'article que nous sommes actuellement en train de réaliser sur les manuscrits 863-864 et 1224 de la bibliothèque de Cambrai. Signalons simplement que nous sommes parvenus à décomposer le ms 863 en 7 parties distinctes, correspondant aux 7 sources différentes utilisées pour sa conception. Les parties I (folios 2 à 49) et 3 (folios 61 à 189), tirées de deux lectionnaires, et la partie 5 (folios 200 à 254), constituée par Fulbert peccator à partir d'un recueil de vies de saints, présentent de très grandes similitudes avec le contenu du ms 1224, visiblement constitué uniquement à partir de ces 3 mêmes sources.

53. Ce lectionnaire est celui qui a permis de constituer la partie 3 (folios 61 à 189) du ms 863 .

54. PithOU, 1687, p. 368 ; SOT, 1993, p. 18; Manuscrits datés des bibliothèques de France, 2000, t. I (Cambrai), p. 87 : les régions d'Arras et de Cambrai, évangélisées par saint Vaast (+540) sous saint Remi, formaient d'abord un évêché administré depuis Arras. Sous saint Géry (584/590-apr. 624), ou son prédécesseur Welulfus (545-584/590), l'administration a été transférée d'Arras à Cambrai. Les deux évêchés sont restés liés plusieurs siècles avant d'être séparés seulement en 1093-1094.

55. Histoire littéraire de la France, 1867, t. VI, p. 40 ; PITHOU, 1687, p. 367-368. L'existence du manuscrit de Cambrai 854, dont la première partie copiée au $\mathrm{X}^{\mathrm{e}}$ siècle correspond à un lectionnaire respectant la chronologie du calendrier des vies de saints apôtres, semble confirmer la mise en place de l'ordre de ces deux églises plutôt sur la fin du Xe siècle qu'au commencement du XI ${ }^{\mathrm{e}}$.

56. Gallia Christiana, 1876, t. III, p. 14-22; Histoire littéraire de la France, 1867, t. VI, p. 31. 
place certaines coutumes issues de leur lieu de formation. Ainsi, le premier lectionnaire, constitué de vies de saints apôtres, s'est vu ajouter, par rapport à l'exemplaire "primitif», des vies de saints sans doute fournies par des manuscrits d'origine liégeoise ${ }^{57}$. De même, le second lectionnaire contenant les vies de martyrs et confesseurs, semble également avoir des liens avec le milieu liégeois, puisqu'on y trouve la Vie de saint Lambert rédigée par Étienne. Plusieurs autres hagiographies présentes dans le recueil du Saint-Sépulcre figurent également dans des manuscrits belges des $\mathrm{X}^{\mathrm{e}}$ et $\mathrm{XI}^{\mathrm{e}}$ siècles $^{58}$. On peut ainsi suggérer la composition des deux lectionnaires entre la fin du $\mathrm{X}^{\mathrm{e}}$ et le début du XI ${ }^{\mathrm{e}}$ siècle, sous les épiscopats de Rothard et Herluin, c'est-àdire entre 976 et $1012^{59}$. Or cette période est contemporaine de Dudon. Le culte porté à saint Lambert sur Cambrai défie ensuite les siècles: le saint liégeois est cité dans les litanies et figure toujours dans les lectionnaires composés aux XIV et $\mathrm{XV}^{\mathrm{e}}$ siècles $^{60}$.

Avant de conclure cette partie, remarquons que l'œuvre d'Étienne était également connue du chapitre de Saint-Omer (et peut-être également de l'abbaye de SithiuSaint-Bertin), au plus tard au XII ${ }^{\mathrm{e}}$ siècle. Le ms Saint-Omer BP 715 est un imposant recueil en 4 volumes rassemblant de très nombreuses vies de saints ${ }^{61}$ : la Vita sancti Lamberti apparaît aux folios 98-105v du volume 3, qui appartenait au chapitre de la cathédrale de Saint-Omer. Il est malheureusement extrêmement difficile de déterminer les différentes sources ayant permis de rassembler toutes ces vies de saints.

57. Les vies de saint Philippe (BHL 6814), de saint Bartholomé (BHL 1002), et de saint Thomas (BHL 8136) sont vraisemblablement issues de l'un des manuscrits suivants, ou de l'un de leurs parents: mss Bruxelles KBR 07882 (3188) et 08223 (3193), datant tous deux du XI' siècle. Leurs folios 114-185 et 3-53v contiennent des vies de saints apôtres apparaissant de manière désordonnée, que l'on retrouve de façon ordonnée dans les lectionnaires de Cambrai.

58. Le recueil 863-864 de Cambrai (fin $\mathrm{XI}^{\mathrm{e}}$ ) comporte plusieurs vies de saints que l'on retrouve dans le ms Bruxelles, Bibl. royale 14650-14659 (3236) au Xe siècle (premier manuscrit de notre étude) : saint Remacle (BHL 7113 et 7120), saint Lambert (BHL 4683), saint Leodegarius (BHL 4851) et saint Amand (BHL 0332). Ces saints figurent tous au martyrologe de la cathédrale. On retrouve également ces vies dans le ms 1224 de Cambrai : saint Remacle (BHL 7113), saint Lambert (BHL 4683), saint Leodegarius (BHL 4851). Saint Amand (BHL 0332) ne figure pas dans ce ms et pour cause : il est fêté le 6 février et cette date coïncide avec une des lacunes de ce manuscrit.

59. Il est possible que les mss Cambrai 365 et 546, datés de la fin du $\mathrm{X}^{\mathrm{e}}$ siècle et contenant des homélies sur les évangiles des temps d'hiver et d'été, et donc présentant un certain ordonnancement, aient été constitués pour l'usage du chapitre Notre-Dame de Cambrai à la même époque que les deux lectionnaires disparus : une partie du mobilier liturgique aurait donc été reconstitué à cette époque (voyez Manuscrits datés des bibliothèques de France, 2000, t. I (Cambrai), p. 61 et 77).

60. Saint Lambert est le premier saint cité dans les litanies du ms Cambrai BP 135 et figure dans le lectionnaire Cambrai BP 806. Voyez le Catalogue général des manuscrits des bibliothèques..., 1891, t. XVII (Cambrai), p. 35 et 295.

61. Cf. Catalogue général des manuscrits des départements..., 1861, t. III (Saint-Omer), p. 316-317; ce catalogue se montre peu précis et ne donne aucune information concernant l'œuvre d'Étienne de Liège. Toutefois, celle-ci a été signalée dans ce manuscrit par les Bollandistes, qui ont réalisé l'inventaire des documents hagiographiques conservés à Saint-Omer. Notons que le volume 1 du recueil appartenait à l'abbaye de Saint-Bertin, tandis que les trois suivants étaient en possession du chapitre de la cathédrale de Saint-Omer; un grand nombre de vies présentes dans le volume 1 se retrouvent d'ailleurs dans les trois autres volumes: il est possible qu'il ait appartenu à un autre recueil copié sur celui du chapitre de la cathédrale (ou inversement). 
A priori, on retrouve dans ce recueil de nombreuses vies également présentes dans des manuscrits issus des diocèses voisins d'Arras, de Cambrai et de Liège. Il semble avoir été élaboré pour regrouper ensemble un maximum de vies de saints, en les réunissant par grandes périodes liturgiques matérialisées par des volumes distincts.

Il paraît inutile de poursuivre plus loin notre étude: les autres manuscrits renfermant la Vie de saint Lambert d'Étienne de Liège sont tous tardifs et semblent originaires de lieux situés plus à l'est, essentiellement dans l'Empire germanique, donc loin des centres ayant pu influencer directement Dudon de Saint-Quentin autour de l'an mil: ils appartiennent aujourd'hui à des bibliothèques de Belgique, d'Allemagne, d'Autriche, des Pays-Bas et d'Italie ${ }^{62}$. En nous concentrant sur le royaume de France et le diocèse de Liège, nous pouvons à présent dresser une carte représentant la zone occidentale de diffusion de l'œuvre d'Étienne (cf. carte 1$)^{63}$.

Si l'œuvre d'Étienne a bien été composée à Liège au début du $\mathrm{X}^{\mathrm{e}}$ siècle, elle s'est visiblement propagée en dehors de cette cité dans les années qui suivirent et autour de l'an mil. Ainsi, Dudon aurait pu en prendre connaissance sans s'être rendu personnellement à Liège.

\section{La Vita sancti Germani du moine Heiric d'Auxerre}

Les emprunts du chanoine de Saint-Quentin à la Vita sancti Germani d'Heiric d'Auxerre semblent avant tout d'ordre stylistique. Leah Shopkow a montré que Dudon de Saint-Quentin avait cité certains extraits d'auteurs classiques par l'intermédiaire de la Vita d'Heiric ${ }^{64}$. Mais ce sont surtout la structure poétique ${ }^{65}$ et le caractère

62. Voici la liste des manuscrits non pris en compte dans cette étude (nous n'avons malheureusement pas pu vérifier si certains d'entre eux étaient d'origine occidentale par rapport à Liège; une identification de l'origine des deux manuscrits du Vatican pourrait s'avérer instructive) : les mss Vatican lat. 8565 et Trêves BA 93 (102), datant de la seconde moitié du XI ${ }^{\mathrm{e}}$ ou de la première moitié du XII siècle. Le ms Trêves BA 93 (102), du XI et XII ${ }^{\mathrm{e}}$ siècle. Les mss Bruxelles KBR 9742, KBR 19385 (VDG 3253) et MB 5 du XII e siècle. Les mss Vatican Reg. lat 542 et Naples BN codex VIII. B. 51, également du XII ${ }^{\mathrm{e}}$ siècle. Le ms Heiligenkreuz SB 13 de la fin du XII ${ }^{\mathrm{e}}$ siècle. Le ms Würzburg UB MP. TH. F. 125, de la fin du XII ${ }^{e}$ ou du début du XIII' siècle. Les mss Bruxelles KBR 1150-11555 (3233), KBR 7482 (3180) et KBR 7483-7486 (3181) du XIII siècle. Le ms Lilienfeld SB 60, lui aussi du

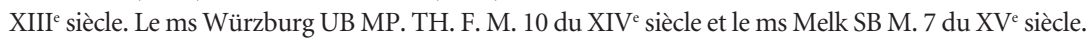

63. Cette carte s'appuie notamment sur « les voies romaines autour de Reims» établies par Michel Sot (SOT, 1993, p. 40, carte $\mathrm{n}^{\circ} 6$ ).

64. SHOPKOW, 1989, p. 23 col. 1-2, p. 25 col. 2 : certaines phrases de l'œuvre de Dudon faisant écho à Horace (Epitres) et Virgile (Enéide) sont en fait des extraits de la Vita sancti Germani d'Heiric. Les traces d'Erigénisme apparaissant chez Dudon dans son dialogue sur la Trinité sont également issues de cette source : c'est donc Heiric qui utilise directement l'œuvre de Jean Scot et non Dudon. L'emprunt fait par le chanoine provient de l'Invocatio présente dans la Vita sancti Germani.

65. Dudon, comme Heiric, intercale plusieurs pièces lyriques au milieu de son texte, pour mettre en valeur les moments forts de son récit (BOUET, 2002, p. 58). L'influence de la Vita sancti Germani se fait également ressentir par l'utilisation de mètres rares, à la manière d'Heiric. Le chanoine de Saint-Quentin reprend d'ailleurs la structure métrique originale de la préface du livre 4, inventée par cet auteur (SHOPKOW, 1989, p. 34 n. 9; JACOBSEN, 1991, p. 335). 
virgilien ${ }^{66}$ de l'œuvre du moine d'Auxerre qui ont intéressé et inspiré le chanoine. Dudon a également exploité abondamment les termes grecs présents dans l'œuvre d'Heiric, en les reproduisant souvent tels quels ${ }^{67}$. De plus, il semble que Dudon ait aussi connu les Miracula sancti Germani d'Heiric, faisant suite à cette Vita: dans le dialogue entre l'abbé Martin et Guillaume Longue-Épée, un passage relatif à la Trinité et à la théorie des trois ordres fonctionnels pourrait fort bien être un remaniement conçu par le chanoine à partir de plusieurs œuvres, dont celle d'Heiric ${ }^{68}$. Ainsi, l'influence de la Vita et des Miracula d'Heiric sur l'œuvre de Dudon fut assez considérable: nous ne la détaillerons pas ici, mais nous reviendrons ultérieurement sur le sujet, dans le cadre d'une étude spécifique.

\section{Heiric et l'école d'Auxerre ${ }^{69}$}

Au moins quatre grands maîtres se sont succédé au cours du IX siècle, entre 835/ 840 et 893 , à la tête de l'école monastique de Saint-Germain d'Auxerre ${ }^{70}$ : Murethach,

66. Heiric semble bien le plus virgilien des poètes du IX siècle (TILliETTE, 1991, p. 321-323). Que Dudon ait choisi ce poète comme modèle dans ce contexte n'est probablement pas une coïncidence: on sait la part tenue par l'Enéide de Virgile dans son œuvre (BOUET, 1990, p. 215-236).

67. JeAUnEAU, 1991, p. 358 et 359-360. Leah Shopkow indique que la totalité des termes grecs utilisés par Dudon provient de la Vita sancti Germani d'Heiric. Ce texte était parfois glosé dans les manuscrits pour expliquer aux non-hellénistes les expressions grecques employées : Dudon aurait pu comprendre certains mots grecs de cette façon (SHOPKOW, 1989, p. 25 col. 2 et p. 34 n. 10). Mais comment pouvait-il comprendre les autres, ceux qui n'étaient pas expliqués dans les gloses, et qu'il utilise malgré tout? Eut-il recours à des lexiques de traduction grec-latin ? Ce genre de lexique était disponible à Laon dès le IX ${ }^{\mathrm{e}}$ siècle: Martin Scot a composé un recueil étymologique que l'on trouve dans le célèbre manuscrit 444 de la Bibliothèque municipale de Laon: Graca collecta ex Prisciano (Jeauneau, 1991, p. 361 ; SOT, 1993, p. 59). Ce type d'outils permettait à des personnes ne lisant pas le grec de traduire certains mots et de les comprendre. Les termes grecs utilisés par Dudon sont ceux qu'Heiric d'Auxerre a lui-même puisés chez Jean Scot (Periphyseon et Carmina érigéniens tel que le poème Si uis OYPANIAC) et que Martin de Laon a fait figurer dans ses lexiques et glossaires de traduction grec-latin : la source initiale est donc la même chez Dudon, Heiric et Martin de Laon, puisqu'elle remonte aux œuvres de l'Erigène.

68. Cet épisode apparaît, sans être développé, dans la Complainte sur la mort de Guillaume LongueÉpée, rédigée peu après 942 : Dudon le connait soit sous cette forme, soit sous la forme d'une Vita Guillelmi composée vers la même époque, et depuis perdue (LE MAHO, 2001, p. 11-32). Le schéma des trois ordres proposé par Dudon differe de celui d'Heiric (bien qu'il utilise des termes grecques que l'on retrouve chez cet auteur), et se rapproche davantage de la conception proposée dans la Vie de saint Hugues de Jumièges: Dudon a peut-être proposé une version personnalisée du schéma des trois ordres en remaniant ceux définis dans les Miracula sancti Germani, dans la Vita prima Hugonis et dans les commentaires bibliques très répandus d'Haimon d'Auxerre.

69. L'école carolingienne d'Auxerre a été étudiée avec un soin tout particulier par un groupe de recherche du CNRS à la fin du siècle dernier. Deux ouvrages constituent depuis la référence pour ces travaux. Tout d'abord, le catalogue de l'exposition: Saint-Germain d'Auxerre, intellectuels et artistes dans l'Europe carolingienne, IX $X^{e}-X I^{e}$ siècles. Ensuite, L'école carolingienne d'Auxerre, de Murethach à Remi (830-908). L'essentiel des références relatives à l'école d'Auxerre dans cet article porte sur les contributions des différents auteurs étant intervenus dans ces ouvrages collectifs. Voir aussi Dictionnaire du Moyen Âge, 2002, p. 115-117 et Dictionnaire des lettres françaises, 1964, p. 370.

70. "Avant-propos ", in L'école d'Auxerre..., 1991, p. 6; JEUDY, 1990, p. 32 ; HoLTZ, 1991, p. 131-132; SASSIER, 1991, p. 32-34 et 35 n 39; IOGNA-PrAT, 1990, p. 277. Saint-Germain est une abbaye royale 
Haymon, Heiric et Remi ${ }^{71}$. Cette succession de maîtres et d'élèves, devenant maîtres à leur tour, a peut-être même débuté plus tôt: Loup de Ferrières (v. 805-862), dont la famille d'origine bavaroise était apparentée à plusieurs évêques d'Auxerre, fut certainement l'un des fondateurs de cette école aux côtés de Murethach, et il forma personnellement plusieurs moines de cette abbaye ${ }^{72}$. Tout au long de cette période, l'école est en relation constante avec la plupart des autres grands centres carolingiens, ce qui se traduit par une circulation d'élèves et par des échanges de manuscrits entre ces différents foyers culturels ${ }^{73}$. Mais c'est au troisième des grands maitres auxerrois que nous allons nous intéresser en particulier, puisqu'il est l'auteur d'une œuvre hagiographique connue de Dudon de Saint-Quentin.

Heiric $^{74}$ est né en 841, mais on ignore son lieu de naissance exact. Offert par ses parents comme oblat au monastère de Saint-Germain d'Auxerre vers 848, il était probablement originaire de Bourgogne, d'un lieu proche d'Auxerre (voire peut-être même de cette cité). Tonsuré à Noël 850, il accède ainsi à la condition monastique. Son premier maître, Haymon, le forme surtout aux «sciences divines $~^{75}$, mais il

depuis Pépin le Bref (751-768). Mais c'est au moment de la renaissance carolingienne, et surtout sous le règne de Charles le Chauve (840-877) - et vraisemblablement grâce à l'influence de ce roi que l'activité littéraire d'Auxerre fut la plus brillante. Les maîtres d'Auxerre, en particulier Haymon et Heiric, sont sollicités par le roi et son entourage laïc pour intégrer les groupes savants débattant des grandes questions théologiques de l'époque, aux côtés des grands intellectuels palatins que sont Loup Servat et Jean Scot. Deux des fils du roi, Lothaire et Carloman, occupent d'ailleurs la tête de l'abbaye entre 863 et 869 . Dans l'ordre des sanctuaires du royaume, Saint-Germain apparait alors au troisième rang des bénéficiaires derrière les deux établissements les plus proches du trône que sont Saint-Denis de Paris et Saint-Martin de Tours. Après la mort de Charles, dernier grand Carolingien, en 877, la présence directe de la royauté à Auxerre disparaît au profit des premiers ducs bourguignons (Richard le Justicier et ses successeurs) : une époque de déclin de l'abbaye s'amorce dès lors, jusqu'à la renaissance due aux moines clunisiens à la fin du $\mathrm{X}^{\mathrm{e}}$ siècle.

71. Haymon fut l'élève de Murethach, auquel il succède vers 840 ; Heiric fut l'élève d'Haymon, qu'il remplace vers 865 ; Remi fut l'élève d'Heiric (et peut-être également d'Haymon) : il prend à son tour la tête de l'école d'Auxerre après 875.

72. SoT, 1993, p. 61 ; HolTZ, 1991, p. 133. Heiric et Remi eurent tous deux Loup Servat, abbé de Ferrières, comme maître. Ce dernier, dont la famille bavaroise était implantée dans la région d'Auxerre depuis l'époque de Charles Martel, était apparenté aux évêques de cette cité: Angelhelme, Héribald (828-857) et Abbon (857-860). Il était sans doute également parent avec son élève Remi d'Auxerre.

73. Parmi ces centres intellectuels, citons Ferrières-en-Gâtinais, Fleury-sur-Loire, Laon, Reims, Lyon et même Fulda en Francie orientale. Les relations avec les savants de la cour palatine de Charles le Chauve semblent permanentes. Saint-Amand est également en relation avec Auxerre grâce à Hucbald, qui suit les cours d'Heiric aux côtés de Remi, avant d'aller enseigner à Reims en compagnie de ce dernier; Hucbald avait entre-temps enseigné à Saint-Amand (Elnones) et à Saint-Bertin (Sithiu). Cf. LOBRICHON, 1991, p. 63 ; HoLTZ, 1991, p. 141 ; SAPIN, 1990, p. 229-232; SOT, 1993, p. 65.

74. La biographie d'Heiric d'Auxerre est assez bien connue: elle a pu être définie en partie grâce aux notes autographes qu'il nous a laissées dans un calendrier figurant dans le ms Melk, Stiftsbibliothek 412 (370/G 32), ainsi que par divers indices apparaissant dans ses œuvres, notamment dans ses Collectanea (QUADRI, 1990, p. 37-40; QUADRI, 1990, p. 41-42).

75. Haymon, qui reçut l'enseignement du premier maître de l'école d'Auxerre, le grammairien d'origine irlandaise Murethach, devient à son tour maître de cette école vers 840 . Sa période d'activité, qui débute à cette date, se poursuit jusqu'en 860 . Grand commentateur de la Bible, son enseignement repose sur des leçons exégétiques et biblico-théologiques. Heiric semble avoir suivi ses cours au moins 
reçoit également des leçons d'humanités de la part de l'abbé Loup de Ferrières ${ }^{76}$. Heiric est ordonné sous-diacre en $859^{77}$, avant de devenir prêtre en $865^{78}$. Entre ces deux dates, sa vie est plus mal connue. Il a probablement séjourné à Soissons ${ }^{79}$ entre 862 et 865 , où il semble être entré en contact avec le cercle érigénien par l'intermédiaire de l'abbé Wulfade de Saint-Médard, futur archevêque de Bourges (866-876) ${ }^{80}$. Toutefois, Heiric a aussi pu accompagner ce même Wulfade à la cour de Laon ${ }^{81}$. Quoi qu'il en soit, c'est après la nomination de Lothaire, fils de Charles le Chauve, comme abbé laïc de Saint-Germain d'Auxerre en $863^{82}$, qu'Heiric entreprend, peut-être à l'instigation de cet abbé, l'adaptation en vers de la Vie de saint Germain initialement composée en prose par Constance de Lyon ${ }^{83}$. Ce travail conséquent débute après 865 et prend fin vers 873/874, époque à laquelle il dédicace son œuvre à Charles le

jusqu'en 859 lorsqu'il est ordonné sous-diacre. Haymon devient abbé de Cessy-les-Bois, probablement entre 865 et 875 , et il occupe vraisemblablement cette place au moment de sa mort (IOGNA-PRAT, 1990, p. 36 ; sur la méthode d'exégèse d'Haymon, voir OrTIGUES et IOGNA-PRAT, 1990, p. 62).

76. Les cours dispensés par Loup Servat à Heiric avaient probablement lieu à Ferrières-en-Gatinais, où Loup fut abbé (841-862). Le moine d'Auxerre semble avoir suivi les leçons de ce maître peut-être jusqu'au décès de celui-ci, en 862. Avec Loup, élève d'Aldric à Ferrières (avant 828) et de Raban Maur à Fulda (de 828 à 836), eux-mêmes tous deux formés par Alcuin (abbé de Ferrières et de Saint-Martin de Tours), Heiric reçoit une culture profane et une connaissance des doctrines d'Alcuin (HOLTZ, 1991, p. 131, 142 n 4 ; RICHÉ, 1979, p. 107; Dictionnaire des biographies, 1993, p. 214-215). Dans le ms London, British Library, Harley 2735, qui contient le Liber Glossarum, sorte de dictionnaire étymologique constitué à la cour de Charlemagne, des additions de la main d'Heiric montrent les influences classiques de Loup sur son élève (GANZ, 1990, p. 42-43). Ces influences transparaissent également dans les Collectanea d'Heiric.

77. Le 22 septembre (10 des calendes d'octobre).

78. La veille des calendes d'avril 865 . 56 jours après son ordination (c'est-à-dire, le 8 des calendes de juin 865), il est invité à revenir au cloître: Heiric semble être présent à Saint-Germain d'Auxerre en tant qu'écolâtre à partir de cette date (il succède ainsi à Haymon, devenu abbé de Cessy-les-Bois vers cette époque), et au moins jusqu'en 875 , année où l'on perd sa trace.

79. HoltZ, 1991, p. 137. Les liens d'Heiric avec Soissons transparaissent à plusieurs niveaux: dans le calendrier annoté de sa propre main, plusieurs inscriptions ainsi qu'une liste de saints concernent directement cette cité ; certaines notices figurant dans les Miracles de saint Germain trahissent une bonne connaissance de cette ville; enfin, il dédia ses collectanea à l'évêque Hildebold de Soissons (871-884).

80. Wulfade/Vulfade appartenait à la cour palatine de Charles le Chauve, où il était en contact avec Jean Scot (dit Erigène), qui lui dédia son œuvre majeure, le Periphyseon. Grand savant, Wulfade laissa pas moins de 31 manuscrits à son archevêché de Bourges, et c'est probablement par son intermédiaire qu'Heiric entra en contact avec l'école hellénisante de Jean Scot (CONTRENI, 1972, p. 923 ; CONTRENI, 1978, p. 145 et suiv. ; IOGNA-PRAT, 1986, p. 119, nº 6).

81. SoT, 1993, p. 62. Heiric entre en contact avec le cercle érigénien à l'époque où Jean Scot finit de rédiger son œuvre principale, le Periphyseon (parfois appelé De divisione naturae), qu'il composa entre 862 et 866 . Lorsqu'il commence la rédaction de sa Vita sancti Germani, sans doute après 865, Heiric vient de découvrir cette œuvre (HOLTZ, 1991, p. 138).

82. Il semble qu'Heiric ait été rappelé à Auxerre à la demande d'une personne ayant une grande autorité: il pourrait s'agir de l'abbé laïc Lothaire, fils infirme de Charles le Chauve, ou de son frère Carloman, qui furent successivement à la tête de l'abbaye de 863 à 866 et de 866 à 869 (SASSIER, 1991, p. 33).

83. Vers 470-480, Constance, écrivain et aristocrate lettré réputé du clergé lyonnais, a écrit à la demande de son évêque Patiens de Lyon la première biographie de saint Germain d'Auxerre (+445/446), environ trente ans après la mort de ce dernier (PICARD, 1990, p. 3 et 7 ; LOBRICHON, 1990, p. 69). 
Chauve $^{84}$. Heiric adjoint à son texte une première version des Miracles de saint Germain en prose et un sermon relatif à ce saint. Son œuvre, mettant en avant l'excellence de la condition monastique par rapport à la condition cléricale, aura pour effet une réplique immédiate de la part des chanoines de la cathédrale d'Auxerre: les Gestes des évêques d'Auxerre, composés entre 873 et 877 à la demande de l'évêque Wala, revendiquent clairement la première place de ces derniers dans la cité ${ }^{85}$. Heiric répondra à son tour en remaniant ses Miracles en prose et en accentuant encore davantage la primauté de l'abbaye Saint-Germain sur la cathédrale ${ }^{86}$. On perd ensuite sa trace vers $875-877^{87}$

La production littéraire d'Heiric se concentre donc sur une dizaine d'années, entre 865 et 875 (ou 877) ${ }^{88}$, et se situe à un carrefour marqué par l'influence de trois grands maîtres carolingiens : Haymon d'Auxerre (formation théologique et exégétique), Loup de Ferrières (formation profane, historique et classique) et Jean Scot (formation philosophique) ${ }^{89}$.

La Vie de saint Germain composée par Heiric n'eut qu'un succès limité : celle composée par Constance de Lyon au $\mathrm{V}^{\mathrm{e}}$ siècle a connu une diffusion beaucoup plus large; c'est elle qui, avec la Vie interpolée de saint Germain, trouve sa place dans la liturgie et les légendiers qui s'intéressent à l'évêque Germain d'Auxerre ${ }^{90}$. Le succès

84. Sur l'identification controversée de l'année de cette dédicace (avant ou après 875), voyez IOGNA-PRAT, 1986, p. 101-102, QUADRI, 1990, p. 37 et 39 et HoLTZ, 1991, p. 153. Nous adoptons ici l'opinion des deux premiers auteurs.

85. Les Gestes des évêques d'Auxerre (Gesta pontificum Autissiodorensium) ont été composés en plusieurs étapes. Le premier tronc, qui nous intéresse ici, a été écrit par deux chanoines de la cathédrale, Alagus et Rainogala, sur l'ordre de leur évêque Wala (872/3-879). Ils constituèrent les 38 premières biographies des évêques d'Auxerre, portant ainsi leurs travaux jusqu'à l'année 872 (LOBRICHON, 1990, p. 286287 ; Les gestes des évêques d'Auxerre, 2002, p. 1 et suiv.).

86. IOGNA-PRAT, 1990, p. 101-102. Les Miracula sancti Germani semblent avoir été constitués en deux ou trois phases.

87. La dernière note d'Heiric dans le calendrier du manuscrit du Melk indique la mort de l'empereur Lothaire en 875 . On remarquera qu'il ne mentionne pas dans son calendrier la mort de l'empereur Charles le Chauve, advenue en 877 , alors que ce dernier tient une place importante dans sa Vita et ses Miracula sancti Germani. La mort d'Heiric serait survenue vers 875/877 selon Riccardo Quadri. Pour John J. Contreni, il faudrait plutôt la placer en 883 (JEUDY, 1990, p. 44).

88. Heiric compose des Collectanea (873-875), un grand homéliaire de 115 sermons, une Homélie sur l'Evangile de Jean, des gloses sur les Categoriae decem du Pseudo-Augustin et sur Priscien, un florilège du Periphyseon de Jean Scot et probablement un recueil de gloses bibliques tirées d'Haymon et de Jean Scot (IOGNA-PRAT, 1990 p. 58-59; QUADRI, 1990, p. 39-40).

89. Jean $\operatorname{Scot}(\sim 800 / 810-\sim 870)$, est le premier auteur qui, dans l'Occident latin, redécouvre la pensée néoplatonicienne. Helléniste, il est le traducteur de plusieurs œuvres d'auteurs grecs, dont les Hiérarchies du Pseudo-Denys l'Aréopagite, les Ambigua et les Quaestiones ad Thalassium de Maxime le Confesseur, qui le mettent en contact avec le néoplatonisme hérité de Proclus et de Damascius. Par l'intermédiaire d'Augustin et de Marius Victorinus, Jean Scot connaît également le néoplatonisme issu de Plotin et de Porphyre (Iogna-Prat, 1990, p. 254 ; Dictionnaire du Moyen Âge, 2002, p. 771-774).

90. D'après Guy Lobrichon, ce succès ne semble être qu'un succès d'école : le monachisme « agressif » d'Heiric ne paraît pas avoir trouvé sa place dans les offices religieux (LOBRICHON, 1990, p. 69). Les Bollandistes ont recensé une centaine de manuscrits contenant l'œuvre en prose de Constance de 
des Miracula sancti Germani fut semble-t-il un peu plus important ${ }^{91}$. Et bien que ces deux œuvres aient été initialement réunies par leur auteur dans un même manuscrit dédié à Charles le Chauve, leur style différent, l'un en vers, l'autre en prose, a probablement été la cause de leur séparation rapide, et de leur diffusion parallèle par la suite $^{92}$. Toutefois, malgré cette propagation a priori assez limitée, la théologie politique apparaissant dans l'œuvre d'Heiric connut à la fois un vif succès - auprès des moines proches de Cluny - et une vive opposition chez les évêques soucieux de préserver leurs privilèges. Nous n'avons donc sans doute gardé que quelques uns des nombreux manuscrits des Miracles de saint Germain.

Nous n'étudierons pas ici en détail la façon dont Heiric a construit son idéologie, ni de quelle manière son texte a pu influencer Dudon de Saint-Quentin : cela nous éloignerait trop de notre sujet initial ${ }^{93}$. Contentons-nous simplement d'indiquer que son œuvre a été utilisée lors des débats politico-théologiques opposant les milieux monastiques et cléricaux autour de l'an mil: les monastères de Fleury ${ }^{94}$ et de Cluny ${ }^{95}$, ainsi que les cathédrales de Laon ${ }^{96}$ et de Cambrai ${ }^{97}$ furent semble-t-il des centres touchés par ces discussions polémiques.

Lyon (BHL 3453, 3454 et 3454b); or, moins d'une dizaine de manuscrits renferme l'œuvre en vers d'Heiric d'Auxerre (BHL 3458).

91. Les Bollandistes ont relevé 15 manuscrits des Miracula (BHL 3462 et 3462b), contre seulement 9 pour la Vita (BHL 3458). Mais une fois encore, ce succès fut surtout un succès d'école (peu de place pour les Miracula dans la liturgie et dans les légendiers).

92. La teneur politique des Miracula, moins présente dans la Vita, pourrait également expliquer la séparation de ces deux œuvres.

93. Nous reviendrons lors d'une étude ultérieure sur ces deux points : Heiric s'appuie en fait sur deux commentaires exégétiques composés par son maître, Haymon d'Auxerre (Commentaire sur l'Apocalypse et Commentaire sur le Cantique des Cantique), et il remanie les schémas tripartites proposés par celui-ci pour en créer un nouveau, favorable aux moines.

94. VEZIN, 1991, p. 58 ; Le livre au Moyen Âge, 2002, p. 46: aux IX et X ${ }^{\mathrm{e}}$ siècles, les scriptoria de SaintGermain d'Auxerre et de l'abbaye de Saint-Benoît-sur-Loire étaient très proches. Abbon de Fleury $(+1004)$, lorsqu'il rédige en 994 une tripartition de l'Église dans son Apologeticus a peut-être connu les Miracula sancti Germani composés par Heiric.

95. OrTigues, 1991, p. 208 ; IOGNA-PRAT, 1990, p. 254 et 278 : la tripartition d'Heiric est reprise mot pour mot par l'auteur du Sermo de beato Maiolo qui composa son œuvre entre 1003 et 1010 sous l'abbé Odilon (994 - 1049). Cet auteur utilise également des extraits du Sermon pour la saint Germain d'Heiric; la Vie de saint Maieul de Syrus (BHL 5177, 5178 et 5179) emprunte de nombreux passages d'inspiration néoplatonicienne et érigénienne présents dans la Vie de saint Germain d'Heiric. Ainsi, la Vita, les Miracula et le Sermo sur saint Germain composés par Heiric étaient tous trois connus de Cluny au début du XI ${ }^{\text {e }}$ siècle: cette abbaye disposait donc vraisemblablement d'un exemplaire manuscrit, aujourd'hui disparu, regroupant ces trois œuvres dans un même recueil, comparable à celui formé par Heiric pour le dédier à Charles le Chauve.

96. L'évêque Adalbéron de Laon, dans son Carmen ad Rotbertum regum, composé vers 1027, semble avoir utiliser les Miracula sancti germani d'Heiric pour constituer sa théorie des trois ordres fonctionnels: nous reviendrons un peu plus loin sur le sujet.

97. Gérard, évêque de Cambrai et d'Arras (1012-1048/1051), propose également une idée de trois ordres fonctionnels, hiérarchisés et solidaires, où ceux qui prient, ceux qui combattent et ceux qui travaillent se soutiennent mutuellement, et où l'on trouve les évêques à la tête de l'Église. Cf. ADALBÉrON DE LAON, 1979, p. CVI-CXV; DUBY, 1978, p. 1 et suiv. 


\section{Les manuscrits de la Vie de saint Germain (Vita sancti Germani) d'Heiric d'Auxerre}

Le plus ancien et meilleur témoin de la Vita d'Heiric est un manuscrit écrit dans la seconde moitié du IX siècle et provenant de Laon (ms Paris BnF latin 13757). Il porte l'ex-libris du XVe siècle de la cathédrale de Laon mais, selon l'abbé Merlette et John J. Contreni, ce manuscrit se trouvait à cet endroit dès la fin du IX ${ }^{\mathrm{e}}$ siècle $^{98}$. Il demeurait visiblement en ce lieu au début du XI siècle, puisque l'évêque Adalbéron de Laon semble s'être servi des Miracula sancti Germani, faisant pendant à la Vita, pour constituer sa tripartition sociale dans son Carmen ad Rotbertum Regem aux alentours de l'année $1027^{99}$. Ce précieux manuscrit a longtemps passé pour un autographe, mais cette hypothèse ne tient plus depuis l'article de Giuseppe Billanovich ${ }^{100}$. Il comporte de nombreuses gloses tirées du Periphyseon de Jean Scot ${ }^{101}$ et il est l'un des témoignages des liens existant entre Auxerre et Laon dans la seconde moitié du IX $^{\mathrm{e}}$ siècle ${ }^{102}$.

C'est incontestablement sur ce type de manuscrit, glosé pour expliquer ou/et traduire les différents termes grecs apparaissant dans le texte, que Dudon s'est appuyé. Le fait qu'il soit l'un des rares - sans doute le seul - manuscrits conservés comportant à la fois la Vita et les Miracula composés par Heiric forme un argument de poids pour voir en cet exemplaire celui connu de Dudon. Puisque l'évêque Adalbéron de Laon, proche de Dudon, a lui-même utilisé ce manuscrit, cette hypothèse prend encore plus de crédit.

On connait également deux autres manuscrits anciens, antérieurs au XI siècle, mais tous deux sont incomplets, et aucun n'est accompagné des Miracula: le premier, datant du IX ${ }^{\mathrm{e}}$ ou X ${ }^{\mathrm{e}}$ siècle, provient de l'abbaye de Saint-Benoît-sur-Loire, dite abbaye

98. HOLTZ, 1991, p. 138; IOGNA-PRAT, 1986, p. 119 n 9 et p. 126 n $^{\circ} 128$; CONTRENI, 1972, p. 37 n 1 ; CONTRENI, 1978, p. 149-150; JeAunEaU, 1991, p. 356-357 et p. 368 n² 29: le ms Laon BM 469, du $\mathrm{IX}^{\mathrm{e}}$ siècle et originaire de la cathédrale de Laon, contient (au folio 182) des scolies tirées du ms Paris BnF lat. 13757, ce qui indique la présence de ce dernier à Laon dès la fin du IX e siècle.Voir aussi la notice relative au ms 13757 (IOGNA-PRAT, 1990, p. 70) : ce manuscrit comporte la Vie métrique composée par Heiric et dédiée à Charles le Chauve (folios 2 à 87), ainsi que les deux livres des Miracles (folios 88 à 151). Enfin, le sermon sur la fête de saint Germain d'Auxerre est présent en fin de recueil (folios 152 à 155).

99. IOGNA-PRAT, 1986, p. 118.

100. HOLTZ, 1991, p. 138 ; JEAUNEAU, 1991, p. 357 et 368 n² 27. Il s'agit visiblement d’une copie proche de l'original, qui pourrait bien être l'exemplaire personnel d'Heiric: il représente peut-être la mise au net de son manuscrit autographe.

101. JEAUNEAU, 1991, p. 357-358 et 368 n² 27 ; IOGNA-PRAT, 1990 p. 255. Les scholies et gloses, légèrement postérieures à la copie du manuscrit, paraissent être de quatre mains différentes sans qu'aucune ne puisse être identifiée à celle d'Heiric. Elles semblent tirées de la troisième recension du Periphyseon, telle qu'on peut la trouver dans les mss Paris BnF lat. 12960 et 12964 . Voyez aussi HoLTZ, 1991, p. 137.

102. HoLTZ, 1991, p. 138 : à l'époque d'Heiric, «il a existé des liens étroits entre Auxerre et Laon, comme le suggère la circulation des manuscrits ». Voir aussi GANZ, 1990, p. 298, 301 et 302 ; IOGNA-PRAT, 1991, p. 164. 
de Fleury (ms Paris BnF latin $6400 \mathrm{~B})^{103}$. Cette abbaye était en étroite relation avec Auxerre, à tel point qu'il est souvent difficile de différencier les manuscrits provenant de l'un ou l'autre scriptorium. Le second, du X $\mathrm{X}^{\mathrm{e}}$ siècle, provient de Saint-Corneille de Compiègne (ms Paris BnF latin 17302). Ce manuscrit, avec celui de Laon, appartenait donc à un lieu proche du pouvoir royal : Laon et Compiègne, avec Quierzy, ont en effet la particularité d'être des cités disposant de palais royaux, donc accueillant la cour, aux IX $\mathrm{X}^{\mathrm{e}}$ et $\mathrm{X}^{\mathrm{e}}$ siècles ${ }^{104}$.

Plusieurs autres manuscrits plus récents nous sont également parvenus, mais ils présentent un intérêt moindre dans le cadre de notre étude: les trois plus anciens sont des $\mathrm{XI}^{\mathrm{e}}$ et XII ${ }^{\mathrm{e}}$ siècles ${ }^{105}$. On remarquera également une famille plus récente de quatre recueils de vies de saints, sans doute d'origine germanique, contenant cette œuvre ${ }^{106}$

Nous pouvons à présent dresser une carte représentant les lieux où la présence de la Vita sancti Germani et des Miracula sancti Germani d'Heiric d'Auxerre est attestée à l'époque de Dudon de Saint-Quentin, c'est-à-dire entre la fin du $\mathrm{X}^{\mathrm{e}}$ et le début du XI ${ }^{\mathrm{e}}$ siècle (cf. carte 2 ).

Ainsi, de même qu'il n'était pas nécessaire d'avoir étudié à Liège à la fin du $\mathrm{X}^{\mathrm{e}}$ siècle pour connaître la Vita sancti Lamberti d'Étienne de Liège, il n'était pas indispensable d'avoir été formé à Auxerre à cette même époque pour connaître la Vita sancti Germani d'Heiric d'Auxerre. Plusieurs indices laissent penser que Dudon a pu connaître la Vita sancti Germani et les Miracula sancti Germani ${ }^{107}$ d'Heiric par l'intermédiaire du manuscrit présent à Laon dès la fin du IX $\mathrm{X}^{\mathrm{e}}$ siècle : le ms Paris BnF latin 13757.

\section{L'anonyme Carmen de sancto Cassiano ${ }^{108}$}

Ce poème sur l'évêque saint Cassien d'Autun (Cassianus ep. Hortanus, dein Augustodunensis, BHL 1634, fêté le 5 août) a été composé dans les milieux scots de

103. JEAUNEAU, 1991, p. 358-359. Le manuscrit 6400 B contient une scholie d'influence érigénienne sur le vers 28 de l'Inuocatio qui n'apparaît pas dans le ms 13757, ce qui voudrait dire que ce dernier manuscrit ne transmet pas l'intégralité des gloses ajoutées par Heiric dans le manuscrit autographe de sa Vita sancti Germani.

104. SOT, 1993, p. 59. Voir aussi JeAUneaU, 1991, p. 368, nº 33.

105. JEAUNEAU, 1991, p. 357-358. Ces trois manuscrits anciens sont postérieurs à l'époque où Dudon fut formé; il s'agit du ms Vatican Reg. lat. 1964 du XI ${ }^{\mathrm{e}}$ siècle (son origine serait malgré tout intéressante à déterminer), du ms Cologne (Köln) HA, W. 163, de la seconde moitié du XI ${ }^{e}$ ou de la première moitié du XII ${ }^{e}$ siècle et du ms Oxford, Bodleian Library, Bodley 793 (2641) du XII siècle (non référencé par les Bollandistes).

106. Il s'agit du ms Heiligenkreuz SB 12 (du dernier quart du XII e siècle), des mss Admont SB 24 et Wien ONB 336 (du XIII ${ }^{\mathrm{e}}$ siècle), et du ms Melk SB M. 5 (de la seconde moitié du XV ${ }^{\mathrm{e}}$ siècle).

107. Pour connaître les quinze manuscrits renfermant les Miracula référencés par les Bollandistes, nous vous invitons à consulter le site BHLms, dont l'adresse est précisée en fin de bibliographie.

108. «Carmen de sancto Cassiono», in MGH, Poetae latini aevi Carolini, 1899, p. 181-196. 
Laon, dans le dernier tiers du IX ${ }^{\mathrm{e}}$ siècle ${ }^{109}$. Sans pouvoir véritablement parler de colonie irlandaise à Laon, il semble avoir malgré tout existé une tradition irlandaise de longue date dans cette cité. L'évêché de Laon fut constitué par l'évêque Remi de Reims au $\mathrm{V}^{\mathrm{e}}$ siècle, et dès le siècle suivant, des ermites irlandais venus s'installer dans les espaces déserts environnants se joignirent aux prélats de la région pour développer rapidement le christianisme dans la cité de Laon et les campagnes avoisinantes. L'influence irlandaise et colombanienne est alors très nette, puisque les premiers monastères édifiés vers cette époque sont soumis à la règle de saint Colomban et fonctionnent à l'irlandaise ${ }^{110}$.

Mais c'est surtout suite à la renaissance carolingienne et à partir du milieu du IX ${ }^{\mathrm{e}}$ siècle, sous les évêques Pardule (848-856) et Hincmar le Jeune (858-871) que cette influence semble la plus remarquable ${ }^{111}$. Elle ne fut toutefois pas la seule et on trouve plusieurs générations successives de scolastici ${ }^{112}$ liées à la fois à la cour palatine de Charles le Chauve ${ }^{113}$, à la cathédrale de Laon et à l'abbaye colombanienne de SaintVincent de Laon ${ }^{114}$. La première, irlandaise et couvrant approximativement la période 850-875, était constituée de Jean Scot et surtout de Martin Scot (819-875) ${ }^{115}$. La

109. Ibidem, p. 178-231 (en particulier p. 180); TillietTe, 1991, p. 319. Tout comme le Carmen de sancto Quintino (ms Paris BnF lat. 14143, folios 74-82v d'une main du $\mathrm{IX}^{\mathrm{e}}$ ou $\mathrm{X}^{\mathrm{e}}$ siècle) et le Carmen de sancta Benedicta ( $\mathrm{ms}$ Paris BnF lat. 8431, folios 5-20 d'une main du IX ${ }^{\mathrm{e}}$ siècle), le Carmen de sancto Cassiano semble avoir été constitué dans les milieux irlandais présents à Laon à partir du milieu du IX ${ }^{\mathrm{e}}$ siècle. Du fait des liens de Dudon avec Laon, il serait sans doute intéressant d'entreprendre une étude visant à déterminer une influence éventuelle des deux vies laonnoises de sainte Bénédicte et de saint Quentin sur l'œuvre de ce chanoine.

110. SAINT-DENIS, Alain, p. 51 et 54. LUSSE, 1922, p. 199-203, 208-210, 241, 248-249 et 330-331. À l'époque mérovingienne, vers la fin du VII ${ }^{\mathrm{e}}$ siècle, Laon disposait déjà d'un scriptorium dans l'abbaye NotreDame-Saint-Julien (dont les archives ont malheureusement brûlé en 1112), et ses écoles semblent réputées sous l'évêque Madalgaire.

111. Pardule intervint notamment aux côtés de l'archevêque Hincmar de Reims et de Jean Scot dans les querelles sur la prédestination qui les opposent à «l'hérétique» Godescalc d'Orbais au milieu du IX ${ }^{e}$ siècle.Hincmar le Jeune, neveu homonyme de l'archevêque de Reims, étudia d'abord auprès de son oncle à Reims et composa plusieurs œuvres et traités marqués par une influence irlandaise une fois devenu évêque de Laon. Deux diacres nommés Teutlandus et Hartgarius étaient alors copistes de la cathédrale (CONTRENI, 1972, p. 935).

112. Les Annales de Laon permettent d'établir la liste des écolâtres de Laon à partir du milieu du IX ${ }^{\mathrm{e}}$ siècle (Annales Laudunenses, in MGH SS, 1925, p. 1293-1295; SOT, 1993, p. 59).

113. SAINT-DENIS, 1994, p. 50 et 52-55 et 57 : Laon, tout comme les cités de Quierzy et de Compiègne, dispose d'un palais royal et bénéficie de la présence régulière de la cour itinérante du roi Charles le Chauve. Plus tard, au $\mathrm{X}^{\mathrm{e}}$ siècle et sous les derniers rois carolingiens, Laon joue à la fois le rôle de capitale-forteresse et de grand centre intellectuel et religieux. Les traditions de ce centre culturel issu de la renaissance carolingienne se perpétuèrent tout au long du XI ${ }^{\mathrm{e}}$ siècle.

114. Ibidem, p. 54 : c'est avec la renaissance carolingienne que l'école de Laon, tout comme celle d'Auxerre, prit véritablement son essor au point de fortement venir marquer la culture du IX ${ }^{\mathrm{e}}$ siècle. Dès le début de cette réforme, et tout au long du IX ${ }^{\mathrm{e}}$ siècle, l'église de Laon semble avoir bénéficié d'une succession d'évêques à la fois instruits et désireux de favoriser le développement de leur école; les évêques Gerfrid, Ganelon et leurs successeurs sont en relation avec les maîtres les plus fameux de leur temps: Alcuin, Adalard de Corbie, Angilbert, Jean Scot, etc.

115. Parmi les irlandais ayant laissé des traces de leur présence à Laon du temps de Jean Scot et Martin de Laon (dit l'Hibernien), notons Aldelmus, Fergus, Cathasach, Probus... Mais selon John J. Contreni, 
seconde génération, qui s'étale sur la période 875-900, se distingue par la présence de l'écolâtre Mannon ${ }^{116}$ et du doyen Bernard ${ }^{117}$. L'évêque Didon de Laon joua probablement un rôle actif auprès de ces deux savants ${ }^{118}$. Enfin, une troisième génération $\left(\sim 900\right.$ - 930) est représentée par le doyen puis évêque Adelelm ${ }^{119}$. C’est vraisemblablement sous la seconde génération que fut composé le Carmen de sancto Cassiano.

On notera une persistance de la présence irlandaise au cours du $\mathrm{X}^{\mathrm{e}}$ siècle, puisque sous Roricon (949-976), un moine irlandais du nom de Mac-Allan sera choisi en 961 pour prendre la tête de l'abbaye Saint-Vincent de Laon, puis de l'abbaye SaintMichel-en-Thiérache ${ }^{120}$. C'est peut-être cet abbé, ou l'un de ses compatriotes, qui compose à cette époque et au même endroit le Dialogus de statu sanctae ecclesiae ${ }^{121}$. Ainsi, à partir de la seconde moitié du IX $\mathrm{I}^{\mathrm{e}}$ siècle et au cours du $\mathrm{X}^{\mathrm{e}}$ siècle, l'école de Laon semble avoir été influencée par des traditions diverses, et notamment irlandaises ${ }^{122}$. L'activité de son scriptorium et la renommée de son école cathédrale restèrent importante au moins jusqu'au XI ${ }^{\mathrm{e}}$ siècle ${ }^{123}$.

leur activité ne s'est probablement pas restreinte à ce centre. Seul Martin Scot semble avoir effectué toute sa carrière à Laon (CONTRENI, 1977, p. 59). Les manuscrits utilisés par Jean Scot, Martin Scot et les Irlandais présents à Laon à partir du milieu du IX ${ }^{\mathrm{e}}$ siècle sont essentiellement destinés à l'enseignement (CONTRENI, 1972, p. 931). En se basant sur les dates de naissance figurant dans les Annales de Laon, John J. Contreni pense que Martin Scot a pu être le maître de Mannon et de Bernard, qui devinrent eux-mêmes scolastici de l'école de Laon (CONTRENI, 1977, p. 65). Il indique qu'Hincmar de Laon fut également l'élève et le protecteur de Martin Scot (CONTRENI, 1972, p. 937). Voyez également, RiCHÉ, 1979, p. 91-92, 95, 107, 217-218.

116. Sot, 1993, p. 59: Mannon est défini sous les termes Manno Philosophus à la tête du gymnasium palatinum dans la Vie de saint Radbod. Il semble donc avoir succédé à Jean Scot à la tête de l'école du palais de Charles le Chauve (voir aussi la note $\mathrm{n}^{\circ} 14$ ).

117. SOT, 1993, p. 59; CONTRENI, 1972, p. 929.Bernard (847-903), fut d'abord chanoine et scolasticus de la cathédrale de Laon, avant de devenir doyen du chapitre.

118. Ibidem, p. 923, 937 : l'évêque Didon de Laon (886-895) laissa au moins une vingtaine de manuscrits à la bibliothèque de sa cathédrale : 9 ont survécu ; en comparaison, l'archevêque Hincmar de Reims (845-882) donna au moins 21 manuscrits aux églises de Reims: 19 sont encore conservés aujourd'hui dans la bibliothèque de cette ville.

119. SOT, 1993, p. 59; CONTRENI, 1972, p. 923, 928-929. Adelelm/Alleaume était déjà prieur de la cathédrale en 892. Il succède à Bernard comme doyen (et trésorier) vers 903, puis devient évêque de Laon après Raoul (897-921), de 921 à 930. Bernard et Adelelm ont été longtemps associés à la cathédrale de Laon, où ils ont tous deux enseigné. Ils ont laissé conjointement 23 manuscrits à leur bibliothèque, et Adelelm en ajouta un autre une fois devenu évêque. Il est probable qu'Adelelm fut l'élève de Bernard, et on peut considérer qu'entre 892 et 903 la deuxième et la troisième génération travaillaient ensemble.Voyez aussi JEAUNEAU, 1972, p. 499; CONTRENI, 1978, p. 79; SOT, 1993, p. 58-60.

120. LeCouteuX, 2004, p. 13 et p. $10 \mathrm{n}^{\circ} 36$.

121. SOT, 1993, p. 102 ; LECOUTEUX, 2004, p. 25. Voir aussi les remarques émises par John J. Contreni (1977, p. 66), qui met en doute l'attribution et la datation de cette œuvre, avancées par Heinz Löwe.

122. John J. Contreni (1972, p. 920, 923, 934) a montré que la bibliothèque de Laon a été constituée en grande majorité sur cette période (elle comporte 125 manuscrits des IX ${ }^{\mathrm{e}}$ et $\mathrm{X}^{\mathrm{e}}$ siècles) : Martin, Hincmar, Didon, Bernard, Raoul et Adelelm ont contribué de façon active à la constitution de cette bibliothèque en tant qu'écolâtre, doyen ou évêque sur la période 850-930.

123. Le livre au Moyen Âge, 2002, p. 48, col. 1 : «l'activité du scriptorium de la cathédrale de Laon, dont le nom est resté attaché à un type d'écriture - l'écriture az de Laon -, utilisé au VIII siècle, s'est pour- 
Le Carmen de sancto Cassiano a la particularité d'avoir été écrit en vers léonins : chaque vers est coupé en deux au niveau d'une césure, et les deux hémistiches qui le composent riment ensemble. Il existe donc une contrainte métrique supplémentaire par rapport à la versification classique en hexamètres, et l'emploi des vers léonins était encore peu répandu au IX ${ }^{\mathrm{e}}$ siècle. Leah Shopkow, dans son article, se contente d'indiquer la connaissance de ce texte par Dudon, sans préciser de quelle manière cela se ressent dans l'œuvre de ce dernier ${ }^{124}$.

Le Carmen de sancto Cassiano a connu une diffusion extrêmement réduite (cf. carte 2) : d'une part, ce saint était surtout vénéré localement; d'autre part, le poème ne nous est parvenu que par l'intermédiaire d'un manuscrit unique : le ms Paris BnF lat. $12958^{125}$. Du fait de cette diffusion extrêmement limitée, ce manuscrit ${ }^{126}$ pourrait bien être l'exemplaire connu de Dudon, à moins que notre chanoine ait directement connu le manuscrit autographe de cette œuvre.

\section{Conclusion}

Au terme de cet article, un point rapide au sujet des trois œuvres identifiées par Leah Shopkow comme ayant été utilisées par Dudon de Saint-Quentin s’impose. Tout d'abord, ces trois textes comportent plusieurs similitudes évidentes : ce sont exclusivement des poèmes hagiographiques d'un style littéraire travaillé (versification, poésie lyrique, pièces rimées et rythmées, etc.), au travers desquels apparaît un bel ensemble des différents modèles classiques connus de la renaissance carolingienne $^{127}$. En effet, tous trois sont issus de centres carolingiens importants entre la seconde moitié du $\mathrm{IX}^{\mathrm{e}}$ et la fin du $\mathrm{X}^{\mathrm{e}}$ siècle-Auxerre, Laon et Liège-qui ont d'ailleurs chacun disposé, à un moment ou un autre de cette période, d'une école monastique et d'une école cathédrale réputées. On note cependant un degré d'influence plus ou moins marqué de ces trois œuvres sur l'historiographie de Dudon : la Vie de saint

suivie tout au long du IX ${ }^{\mathrm{e}}$ siècle, et a connu une nouvelle impulsion au XI ${ }^{\mathrm{e}}$ siècle avec la renommée de l'école attachée à la cathédrale».

124. Pour établir les liens entre l'œuvre de Dudon et ce Carmen, il serait intéressant de consulter le texte non publié et composé à Toronto par Leah Shopkow en 1984: «Norman historical writing in eleventh and twelfth centuries".

125. Le Carmen, qui apparaît en fin de manuscrit aux folios 73-76, semble avoir été copié au $\mathrm{X}^{\mathrm{e}}$ ou au $\mathrm{XI}^{\mathrm{e}}$ siècle. Dans son inventaire des manuscrits de la Bibliothèque Nationale de France relatifs aux fonds provenant de l'abbaye Saint-Germain-des-Prés (t. II, p. 82), Léopold Delisle date pourtant l'ensemble de ce manuscrit des $\mathrm{IX}^{\mathrm{e}}$ et $\mathrm{X}^{\mathrm{e}}$ siècles. On remarquera que si ce Carmen a été référencé sous le code BHL 1634 par les Bollandistes, ces derniers n’ont indiqué aucun manuscrit témoin de cette œuvre.

126. Ce recueil regroupe des textes de diverses origines sur le trivium et le quadrivium (dialectique, arithmétique, musique, grammaire, poésie, etc.): il serait intéressant de comparer leur contenu avec la lettre de dédicace de Dudon, où ce dernier traite de sujets aussi divers que d'arithmétique et de musique, comme l'indique Leah Shopkow (SHOPKOW, 1989, p. 23 col. 1). Toutefois, dans l'éventualité de liens étroits entre Dudon et Laon, le chanoine de Saint-Quentin devait probablement connaître le Musica Enchiriadis, composé dans les milieux proches de l'école de Laon, et qui fut l'un des ouvrages de musique ayant connu le plus de succès: il existe plus de 50 témoins manuscrits de cette œuvre (RICHÉ, 1979, p. 275).

127. Leah Shopkow (1989, p. 24, col. 2) précise que la Vie de saint Germain et le Carmen de sancto Cassiano sont des poèmes, tandis que la Vie de saint Lambert est une œuvre en prose métrique (prosimetrum). 
Germain d'Auxerre, composée par Heiric, semble incontestablement le texte qui a le plus influé sur le style de notre auteur ${ }^{128}$; la Vie de saint Lambert, constituée par Étienne, semble avoir elle aussi pesé sur le texte de Dudon, mais peut-être dans une moindre mesure ${ }^{129}$; enfin, le Poème sur saint Cassien d'Autun paraît, a priori, celui des trois textes qui a le moins influencé ce chanoine. Seule une étude linguistique approfondie permettrait de véritablement mesurer l'impact de ces trois poèmes sur le texte de Dudon. Toutefois, la connaissance de ces œuvres trahit la formation de Dudon dans un centre traditionnel de la fin du $\mathrm{X}^{\mathrm{e}}$ siècle s'intéressant de près à la fois à la versification latine et à l'hagiographie. Un lieu qui cherchait visiblement à perpétuer la culture carolingienne de la fin du $\mathrm{IX}^{\mathrm{e}}$ et du début du $\mathrm{X}^{\mathrm{e}}$ siècle, et qui n'avait pas encore été touché en profondeur par les «nouvelles études », telles qu'elles étaient enseignées par Gerbert d'Aurillac à Reims après 970. Plusieurs indices laissent penser que ce pourrait être Laon ${ }^{130}$ :

- Au moins deux des trois œuvres hagiographiques étudiées (les moins répandues) se trouvaient à Laon à la fin du $\mathrm{X}^{\mathrm{e}}$ siècle : la Vita sancti Germani d'Heiric d'Auxerre et le Carmen de sancto Cassiano ${ }^{131}$.

- Les Miracula sancti Germani d'Heiric d'Auxerre, sans doute connus de Dudon, étaient également présents dans cette cité.

- Les Annales de Flodoard, utilisées par Dudon et dont la diffusion fut très limitée, ont vraisemblablement fait l'objet d'une continuation à Laon ${ }^{132}$.

128. Nous avons vu que l'influence d'Heiric sur Dudon se ressent avant tout au niveau du style (emploi de pièces lyriques et d'hexamètres, insertion de pièces de vers dans une œuvre en prose, utilisation de termes grecs dans une œuvre latine). Mais le fond est également concerné (en particulier pour l'érigénisme qui apparaît chez Dudon via Heiric). Dudon paraît également connaître les Miracula sancti Germani d'Heiric (pour l'élaboration, peut-être personnelle, d'un schéma des trois ordres à partir de plusieurs sources).

129. Bien que Dudon ait recours au prosimetrum comme Étienne, le chanoine emploie des rimes généralement plus riches et des poèmes plus longs (ne se réduisant pas à une simple ligne) que ceux présents dans l'œuvre de l'évêque de Liège (SHOPKOW, 1989, p. 27 col. 1 et 35 n 12). Et même si la Vita composée par Heiric d'Auxerre n'adopte pas complètement la structure d'un prosimetrum, les rimes et les poèmes composés par Dudon s’inspirent visiblement davantage de cette œuvre.

130. Bien que formé à Reims par Gerbert d'Aurillac, Adalbéron n’a peut-être pas mis en place «les nouvelles études » à Laon dès son arrivée en 977 et de façon identique à son maître; cela expliquerait peut-être cette impression de «culture mixte» que l'on rencontre chez Dudon : la première, respectant les conventions poétiques mérovingiennes et carolingiennes, probablement encouragée par Roricon; la seconde, ayant recours à la rhétorique, sans pour autant délaisser la poésie et la versification (contrairement à l'enseignement prodigué par Gerbert), que l'on retrouve dans les œuvres d'Adalbéron : les enseignements de Gerbert et d'Adalbéron divergeaient donc très probablement. Adalbéron, arrivé jeune à Laon, fut probablement influencé lui aussi par l'enseignement dispensé dans cette cité (pour l'influence d'Adalbéron sur l'œuvre de Dudon, voir BOUET, 1990, p. 217-218). Dudon, comme Adalbéron, est un grammairien (poète) et un rhéteur plus qu'un dialecticien : sa formation semble davantage marquée par l'école de Laon que celle de Reims, où l'enseignement de la dialectique et de la logique fut restauré par Gerbert (Duby, 1978, p. 65).

131. La troisième, la Vita sancti Lamberti d’Étienne de Liège, était semble-t-il présente non loin de là, à Cambrai et à Arras: Dudon aurait pu la connaître plus tard, après sa formation, et une fois devenu chanoine de la collégiale de Saint-Quentin.

132. LECOUTEUX, 2004, p. 17-22 et 33-34. 
- Enfin, Dudon a dédicacé son œuvre à l'évêque de Laon, Adalbéron, avec lequel il était visiblement très lié : il le tutoie ${ }^{133}$ et le désigne comme le correcteur de son ouvrage ${ }^{134}$. C'est peut-être aussi grâce à ce prélat que Dudon est devenu doyen de la collégiale de Saint-Quentin après 1015.

Comme le montre le tableau 1, indiquant la correspondance entre les lieux de diffusion des œuvres étudiées et les grands centres culturels carolingiens des IX ${ }^{\mathrm{e}}$ et $\mathrm{X}^{\mathrm{e}}$ siècles, c'est à Laon que l'on trouve le plus de traces des textes utilisés par Dudon. Sauf si nous sommes victime des aléas de la conservation ou de la perte de manuscrits, c'est donc bien à Laon, plutôt qu'à Liège ou Reims, que le chanoine de SaintQuentin semble avoir été formé. Le centre carolingien traditionnel ayant principalement et directement influencé Dudon semble donc bien être l'école épiscopale de Laon et le milieu laonnois de l'an mil; même si leur influence ne doit pas être négligée, les milieux rémois, liégeois et auxerrois n'ont visiblement tenu qu'un rôle secondaire et indirect sur la formation et l'œuvre du chanoine de Saint-Quentin ${ }^{135}$.

\begin{tabular}{|c|c|c|c|c|c|}
\hline & 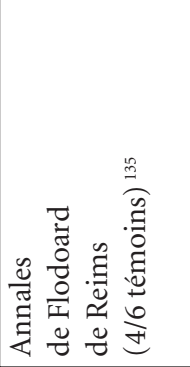 & 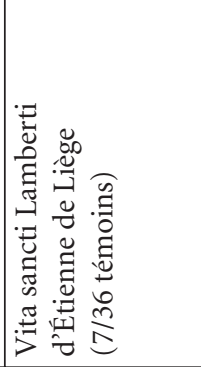 & 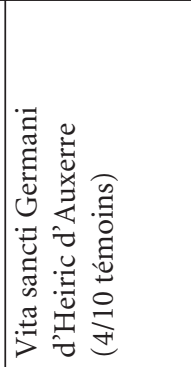 & 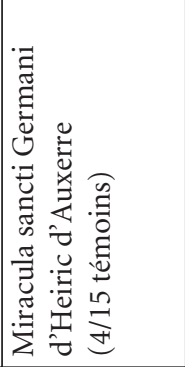 & 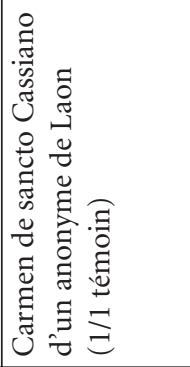 \\
\hline Reims & Lieu d'origine & & & & \\
\hline Liège & & Lieu d'origine & & & \\
\hline Auxerre & & & Lieu d'origine & Lieu d'origine & \\
\hline Laon & $\begin{array}{l}\text { Présence } \\
\text { probable }\end{array}$ & & $\begin{array}{l}\text { Présence } \\
\text { attestée }\end{array}$ & $\begin{array}{l}\text { Présence } \\
\text { attestée }\end{array}$ & Lieu d'origine \\
\hline $\begin{array}{l}\text { Cambrai/ } \\
\text { Arras }\end{array}$ & & $\begin{array}{l}\text { Présence } \\
\text { attestée }\end{array}$ & & $\begin{array}{l}\text { Présence } \\
\text { attestée }\end{array}$ & \\
\hline
\end{tabular}

Tableau 1: correspondance entre les ouvres connues de Dudon et les grands centres carolingiens disposant de ces textes.

Deux points déterminant ressortent clairement de cette étude :

133. DUDON DE SAINT-QUENTIN, 1865, p. 119 et suiv: Dudon utilise le pronom personnel tu sous ses différentes déclinaisons ( $t u i, t i b i, t e)$ en s'adressant à Adalbéron dans sa préface; il n'utilise pas une seule fois vos, ce qui témoigne d'une certaine proximité.

134. Histoire littéraire de la France, 1867, t. VII, p. 237 ; voir aussi BOUET, 1990, p. 217.

135. «4/6 témoins » signifie que sur les 6 témoins connus de cette œuvre, 4 sont antérieurs ou contemporains de Dudon. Bernard Guénée (1980, p. 251) ne comptabilise que 6 manuscrits du Moyen Âge des Annales de Flodoard et non 7 comme Philippe Lauer le fait dans son édition, car le manuscrit d'Albi date du XVII ${ }^{e}$ siècle. 
- Les œuvres nous étant parvenues dans un faible nombre d'exemplaires - de un à quinze exemplaires connus - se trouvaient toutes à Laon (Carmen de sancto Cassiano, Vita sancti Germani, Miracula sancti Germani et probablement Annales de Flodoard); en revanche, on n'en retrouve aucune à Liège et une seule d'entre elles à Reims (les Annales de Flodoard).

- Comment Dudon a-t-il eu connaissance de la Vita sancti Lamberti d'Étienne de Liège, qui est de loin l'œuvre la plus répandue de notre étude, avec près d'une quarantaine d'exemplaires connus? On peut envisager de nombreuses hypothèses: à Laon, dans un manuscrit aujourd'hui perdu; à Liège, voire Cambrai, Arras ou ailleurs, dans le cadre d'un séjour d'étude sans doute plus bref, ou simplement lors d'un court passage ${ }^{136}$.

Si cette étude fait ressortir les liens très étroits que Dudon a entretenus avec Laon, elle ne permet toutefois pas d'affirmer sans réserve que le chanoine a bien été formé à cet endroit dans la seconde moitié du $\mathrm{X}^{\mathrm{e}}$ siècle, et encore moins de préciser si ce fut sous l'évêque Roricon (949-976) ou/et Adalbéron (977-1031): de nombreux indices existent, mais aucun ne fait de cette hypothèse une certitude. En revanche, il semble incontestable que les relations de Dudon avec Laon furent tributaires de la présence de ces deux prélats aux fortes personnalités. Dès lors, une étude du centre culturel et intellectuel de Laon dans la seconde moitié du $\mathrm{X}^{\mathrm{e}}$ siècle est à souhaiter. En effet, si l'on estime généralement que l'activité des écoles de Laon s'est fortement réduite au milieu du $\mathrm{X}^{\mathrm{e}}$ siècle ${ }^{137}$, plusieurs indices suggère que ce jugement est certainement excessif ${ }^{138}$.

D'autre part, il semble possible d'identifier l'auteur du De gestis Francorum avec l'évêque Roricon de Laon ${ }^{139}$. Or, cette œuvre montre de grandes similitudes avec

136. SHOPKOW, 1989, p. 26 col. 1. Les voyages ne rebutaient pas les clercs de l'an mil et un séjour de Dudon à Liège n'est pas à exclure. En effet, avec le prestige dont cette cité jouissait sous l'épiscopat de Notger (972-1008), de nombreux clercs furent attirés à cet endroit pour étudier: si Dudon semble bien avoir été formé principalement à Laon, le suivi d'un enseignement à Liège sur une plus courte période ne parait pas inconcevable. De même, lors de ses déplacements entre Saint-Quentin, Rouen et Fécamp, la proximité de Cambrai et d'Arras sur son parcours permet d'envisager une prise de connaissance de cette œuvre à l'occasion d'un bref passage dans l'une de ces deux cités : l'hébergement de Dudon auprès du chapitre de Cambrai ou d'Arras semble tout à fait concevable. Notre étude irait dans le sens des propos de Georges Duby (Duby, 1978, p. 108), qui souligne que la culture de Dudon «était la même que répandait l'école de Reims et dont les livres conservés à Laon et à Cambrai formaient l'assise».

137. Cette présumée baisse d'activité, intervenue au moment où le monastère de Saint-Vincent développe parallèlement un centre d'enseignement reconnu, est traditionnellement justifiée en se basant sur deux constatations: d'une part, au fait que les évêques Roricon et Adalbéron, qui sont connus comme de savants érudits échangeant avec les intellectuels de leur temps, ne firent jamais mention de l'enseignement dispensé dans leur école cathédrale dans leur correspondance; d'autre part, au plus faible enrichissement des fonds de la bibliothèque laonnoise à partir de cette période (SAINTDENIS, 1994, p. 54-55).

138. Voyez par exemple ADALBÉRON DE LAON, 1979, p. CLI-CLVIII, en particulier CLI et CLIV-CLV; Le livre au Moyen Âge, 2002, p. 48 col. 1.

139. Molinier, 1901, t. 1, p. 66-67 (p. 69 n² 202 pour la liste des éditions); Histoire littéraire de la France, 1867, t. VII, p. 186-187. L'auteur de cette œuvre, qui s'est contenté d'abréger le fameux Gesta regum Francorum composé par un anonyme ( « depuis l'origine de la Nation jusqu'à la mort de 
l'historiographie de Dudon ${ }^{140}$. Les titres initiaux de ces deux œuvres étaient peut-être même apparentés ${ }^{141}$. Ainsi, ces deux textes pourraient sans doute représenter et caractériser la production historiographique laonnoise de la seconde moitié du $\mathrm{X}^{\mathrm{e}}$ et du début du XI ${ }^{\text {e }}$ siècle. Il serait donc intéressant de vérifier les influences du De gestis Francorum ainsi que du Dialogus de statu sanctae ecclesiae ${ }^{142}$, deux œuvres vraisemblablement composées à Laon sous l'évêque Roricon (949-976), sur l'œuvre du chanoine de Saint-Quentin.

Afin d'être complètes et exhaustives, les recherches amorcées dans cet article ne devraient pas s'interrompre ainsi, en se restreignant uniquement à l'étude de quelques-unes des œuvres connues comme étant utilisées par Dudon. Elles devraient se poursuivre sur l'ensemble des textes employés par cet auteur. Ainsi, une liste des principaux lieux susceptibles d'avoir influencé Dudon lors de sa formation ou au cours de sa quête de manuscrits pourrait être établie. De même, la liste des manuscrits utilisés par Dudon pourrait être peu à peu élaborée et enrichie ${ }^{143}$. À terme, l'identification de l'écriture de Dudon, à partir des notes qu'aurait éventuellement pu laisser le chanoine sur certains manuscrits, est peut-être même envisageable. Devant l'ampleur d'une telle démarche, seule la réalisation d'une thèse ou la constitution d'un groupe de travail portant sur ce sujet permettrait de réellement atteindre ces objectifs de façon satisfaisante.

Clovis inclusivement»), s'appelait lui aussi Roricon. Or, bien que ce prénom soit peu répandu, ces deux individus ont été jusqu'à ce jour considérés comme deux auteurs homonymes ayant vécu à des époques différentes. L'auteur du De gestis Francorum a été désigné, sans preuve et vraisemblablement à tord, comme un moine du XI' siècle. Dom Rivet précise qu'il pourrait en fait s'agir d'un clerc ayant rédigé son œuvre dès le $\mathrm{X}^{\mathrm{e}}$ siècle. Ainsi, nous retrouvons toutes les caractéristiques correspondant à notre évêque : identité de prénom, de fonction dans l'Église et d'époque.

140. Nous avons affaire, dans les deux cas, à des poètes-rhéteurs écrivant de l'histoire et laissant la part belle aux légendes, ce qui reste assez peu commun, même dans la seconde moitié du Xe siècle. L'auteur du De gestis Francorum peut-il être identifié avec l'évêque Roricon, qui aurait pu être le premier maître de Dudon à Laon? Le rapprochement entre ces deux auteurs et ces deux œuvres est très séduisant: il serait intéressant d'étudier en profondeur, notamment par une étude linguistique, l'influence éventuelle du De gestis Francorum de Roricon sur le De gestis Normannorum de Dudon.

141. Rappelons que le titre le l'œuvre de Dudon fut peut-être Gesta Normannorum ou Historia Normannorum (BOUET, 2002, p. 58 et LeCOUTEUX, 2004, p. 7). Un titre tel que De gestis Normannorum, se rapprochant fortement du De gestis Francorum de Rociron, serait-il envisageable? Il ne s'agit là que d'une supposition, mais la question mérite d'être posée.

142. Le Dialogus de statu sanctae ecclesiae aurait-il pu fournir à Dudon certains traits relatifs aux rapports entre les églises et les monastères à la fin du $\mathrm{X}^{e}$ siècle?

143. Suite à cette étude, nous pouvons déjà suggérer de faire figurer dans cette liste le ms Paris BnF latin 13757 (renfermant la Vita et les Miracula sancti Germani d'Heiric d'Auxerre) et le ms Paris BnF lat. 12958 (renfermant le Carmen de sancto Cassiano). Le ms Vatican Reg. lat. 6332 (contenant les Annales de Flodoard), contemporain de Dudon et présent à Fécamp, était vraisemblablement aussi connu de cet auteur (même si Dudon utilisa plutôt le manuscrit source de ce dernier pour composer son œuvre). Dans l'hypothèse d'une formation de Dudon à Laon, l'utilisation du ms Laon BP 444 (renfermant un recueil étymologique grec-latin) pour reproduire certains termes grecs inclus dans son œuvre est également à envisager. Il faudrait bien sûr s’intéresser aussi aux 125 mss identifiés par J. Contreni comme appartenant à la Bibliothèque de Laon aux IXe et Xe siècles! Georges Duby indique que la bibliothèque de Laon contenait 300 volumes au $X^{\text {e }}$ siècle (Duby, 1978, p. 86). 
Cela fait aujourd'hui environ un millénaire que Dudon a composé son apologie des «premiers ducs de Normandie», et nous ne connaissons encore que peu de choses sur le premier historiographe normand. Or, les historiens ultérieurs traitant du sujet se sont tous plus ou moins appuyés sur les dires de cet auteur, qu'ils lui aient ou non reproché ses «fables» et ses légendes! Mieux nous connaîtrons Dudon, mieux nous arriverons à saisir son œuvre et les influences qui ont pu s'exercer sur elle. Cette étape semble indispensable pour véritablement comprendre l'historiographie et l'histoire de la Normandie, qui est en grande partie tributaire de Dudon et qui reste relativement obscure pour tout ce qui concerne le $\mathrm{X}^{\mathrm{e}}$ siècle.

\section{Bibliographie $^{144}$}

Adalbéron de LaOn, Poème au roi Robert, éd. et trad. Claude CAROzZI, Paris, Société des Belles-Lettres (Les classiques de l'histoire de France au Moyen Âge, 32), 1979, CLVIII-50 p.

"Annales Laudunenses et sancti Vincentii Mettenses breves », in Monumenta Germaniae historica, Scriptores (MGH SS), Leipzig, W. K. Hiersemann, 1925, t. XV, part. II, p. 1293-1295. (BnF/Gallica).

AudA, Antoine, L'école musicale liégeoise au Xe siècle: Étienne de Liège, Bruxelles, Lamertin, 1923, 211 p.

«Avant-propos», in Intellectuels et artistes..., 1990, p. XVII-XIX.

«Avant-propos », in L'école d'Auxerre..., 1991, p. 5-11.

BAUDUIN, Pierre, La première Normandie ( $X^{e}-X I^{e}$ siècles). Sur les frontières de la haute Normandie: identité et construction d'une principauté, Caen, Presses universitaires de Caen, 2004, 473 p.

BOUET, Pierre, «Dudon de Saint-Quentin et Virgile: L'Enéide au service de la cause normande", in Recueil d'études en hommage à Lucien Musset, Caen, Cahier des Annales de Normandie, n² 23, 1990, p. 215-236.

ID., «Dudon de Saint-Quentin et Fécamp», in Tabularia «Études», n², 2002, p. 57-70.

Catalogue des manuscrits français: anciens fonds (BnF ancien fonds français $\mathrm{n}^{\circ}$ 1-6170), Paris, Didot, 1868-1902, t. I (n 1-3130), IX-783 p.

144. Certains textes sont consultables dans la bibliothèque numérique Gallica de la Bibliothèque nationale de France (dans ce cas, la référence bibliographique est suivie de la mention: BnF/Gallica). Cette bibliothèque est accessible à l'adresse suivante: http://gallica.bnf.fr (utilisez le menu « Recherche»). Pour l'étude des textes hagiographiques, nous avons eu recours à la base de données BHLms (Bibliotheca Hagiographica Latina manuscripta) réalisée par la Société des Bollandistes et le Centre «Hagiographies» (FUNDP). Elle dérive de la base de données «Légendiers » et est accessible gratuitement sur internet à l'adresse suivante : http://bhlms.fltr.ucl.ac.be 
Catalogue général des manuscrits des bibliothèques publiques de France, Paris, Plon, t. I (Rouen, 1886, LVIII-622 p.), t. II (Evreux, 1888, XI-736 p.), t. XVII (Cambrai, 1891 XXIV-593 p.), t. XIX (Amiens, 1893, C-613 p.), t. XXV (Valenciennes, 1894, 637 p.), t. XXXI (Angers, 1898, 825 p.).

Catalogue général des manuscrits des bibliothèques publiques des départements, Paris, Imprimerie impériale, t. I (Laon, 1849, VIII + 904 p.), t. III (Saint-Omer, Saint-Mihiel, 1861, 732 p.), t. IV (Arras, 1872, XII-808 p.), t. VI (Douai, 1878, XII-908 p.).

Catalogue Général des manuscrits français: ancien Saint-Germain français II (BnF fonds français $n^{\circ} 17059-18676$ ), Paris, Leroux, 1898, XVI-517 p.

Catalogue Général des manuscrits français: anciens petits fonds français 8 ( $\mathrm{BnF}$ fonds français $\mathrm{n}^{\circ}$ 22885-25696), Paris, Leroux, 1902.

Catalogus codicum (librorum) manuscriptorum Bibliothecae regiae, Paris, Typographia regia, 1744, t. III et IV (BnF fonds latin $\left.\mathrm{n}^{\circ} 1-8822\right)$.

"Carmen de sancto Cassiono», in Monumenta Germaniae historica, Poetae latini aevi Carolini, Berlin, Weidmannos, 1899, t. IV, p. 178-196.

CHARTIER, Yves, L'œuvre musicale d'Hucbald de Saint-Amand: les compositions et le traité de musique, Montréal, Bellarmin (Cahiers d'études médiévales, cahier spécial $\left.n^{\circ} 5\right), 1995$, XVII-505 p.

ID., «Ambiguïtés de la notation musicale au IX siècle : le (De) Musica (v. 880) d'Hucbald de Saint-Amand (v. 850-930)» in Documents pour servir à l'histoire de la théorie musicale: http://www.musicologie.org/publirem/hmt/ hmt_hucbald.html

ID., «Naissance de la séquence au IX siècle : Notker de Saint-Gall, Préface au Liber Hymnorum (v. 884)» in Documents pour servir à l'histoire de la théorie musicale: http://www.musicologie.org/publirem/hmt/hmt_notker.html

CONTRENI, John J., «À propos de quelques manuscrits de l'École de Laon au IX ${ }^{\mathrm{e}}$ siècle : découvertes et problèmes ", in Le Moyen Âge, $\mathrm{n}^{\circ}$ 1, 1972.

ID., «The formation of Laon's Cathedral Libray», in Studi Medievali, n 13, Spoleto, 1972, p. $919-939$ (réimpr. in the ninth Century, in Carolingian Learning, Masters and Manuscripts, 1992, chap. XIII, p. 919-939).

ID., "The Irish «Colony» at Laon during the time of John Scottus », in Jean Scot Erigène et l'histoire de la philosophie, éd. René ROQUES (Colloques internationaux du CNRS, n 561, Laon, 7-12 juillet 1975), Paris, 1977, p. 59-67 (réimpr. in The ninth Century, in Carolingian Learning, Masters and Manuscripts, 1992, p. 59-67).

ID., "The Cathedral School of Laon from 850 to 930 : its Manuscripts and Masters », in Münchener Beiträge zur Mediäv. U. Renaissance Forschung ( $\left.\mathrm{n}^{\circ} 29\right)$, Munich, 1978. 
Dictionnaire des biographies, Jean-Maurice BIZIÈRE (dir.), Paris, Armand Colin, 1993 (t. II, Le Moyen Âge), 309 p.

Dictionnaire des lettres françaises, Georges DE GRENTE (dir.), Paris, Fayard, 1964 (t. I, Le Moyen Âge), 765 p.

Dictionnaire du Moyen Âge, Claude GAUVARD, Alain DE LIBERA et Michel ZINK (dir.), Paris, Presses Universitaires de France, 2002, LI-1548 p.

DUBY, Georges, Les trois ordres ou l'imaginaire du féodalisme, Paris, Gallimard, 1978, 428 p.

ID., «Préface », in L'école carolingienne d'Auxerre..., 1991, p. 3-4.

DUDON DE SAINT-QUENTIN, De moribus et actis primorum Normanniae ducum, éd. Jules-Auguste LAIR, Caen, le Blanc-Hardel (Mémoires de la Société des Antiquaires de Normandie, 23, $2^{\mathrm{e}}$ partie), 1865, 317 p.

ÉTIENNE DE LIÈGE, «Vita sancti Lamberti», in Patrologiae Latinae (PL), éd. Migne, 1880, t. 132, p. 643-660.

ID., «Excerpta ex Stephani Leodiensis vita sancti Lamberti», in Monumenta Germaniae historica, Poetae latini aevi Carolini, Berlin, Weidmannos, 1899, t. IV, p. 323-233.

FlodoARD, Annales, éd. Philippe LAUER, Paris, Picard (Collection de textes pour servir à l'étude et à l'enseignement de l'histoire), 1906, LXX-307 p.

Gallia Christiana, éd. Paul Piolin, Paris, Palmé, t. III (Arras, Cambrai, SaintOmer, Liège, 1876), t. XIII (Verdun, 1874).

GANZ, David, «Liber Glossarum avec notes de la main d'Heiric d'Auxerre », in Intellectuels et artistes..., 1990, notice $\mathrm{n}^{\circ} 15$, p. 42-44.

ID., «Heiric d'Auxerre glossateur de Liber glossarum », in L'école carolingienne d'Auxerre..., 1991, p. 297-312.

GUÉNÉE, Bernard, Histoire et culture historique dans l'Occident médiéval, Paris, Aubier (collection historique), 1980, 439 pages.

GUÉRIN, Paul, Les petits bollandistes: vies des saints du 10 septembre au 2 octobre, Paris, Bloud et Barral, 1876, t. XI, 656 p. (BnF/Gallica).

«Haymon d'Auxerre, Commentaire sur l'Apocalypse», éd. Dominique IoGNAPrAT et Edmond OrTigUES, in Intellectuels et artistes..., 1990, notice $\mathrm{n}^{\circ} 25$, p. 65-66.

HÉlIN, M. (abbé), Les saints du diocèse de Cambrai, traduction du propre de Cambrai avec courtes réflexions, exemples, prières, Lille, Saint-Charles, 1897, XXVII$523 \mathrm{p}$.

HÉRIGER DE LOBBES, "Gesta episcoporum Tungrensium, Traiectensium et Leodiensium ", in Monumenta Germaniae historica, Scriptores (MGH SS), Leipzig, W. K. Hiersemann, 1925, t. VII, p. 161-189. (BnF/Gallica). 
Histoire de la principauté de Liège: De l'an mille à la Révolution, éd. Bruno DEMOULIN et Jean-Louis KuPPER, Toulouse, Privat (Histoire des provinces), 2002, 271 p.

Histoire Littéraire de la France, éd. Alexis PAUlin, Paris, Palmé, t. IV (1866, XVI719 p.), t. VI (1867, XV-709 p.), t. VII (1867, XCIX-716 p.). (BnF/Gallica).

Holtz, Louis, «L'école d'Auxerre », in L'école carolingienne d'Auxerre..., 1991, p. 131-146.

ID., «Murethach et l'influence de la culture irlandaise à Auxerre », in L'école carolingienne d'Auxerre..., 1991, p. 147-156.

Intellectuels et artistes dans l'Europe carolingienne, IX ${ }^{e}-X I^{e}$ siècles, dir. CNRS, Auxerre, 1990, XIX + 292 p.

Inventaire des manuscrits latins conservés à la bibliothèque nationale (BnF fonds latin n 8823-18613), éd. Léopold DeLISLE, Paris, Durant et Pedone-Lauriel, 1863-1871, t. II ( $\mathrm{n}^{\circ} 11504-14231$ : Saint-Germain-des-Prés) et t. III ( ${ }^{\circ} 14242-$ 15175 : Saint-Victor de Paris).

IOGNA-PRAT, Dominique, "Le "Baptême" du schéma des trois ordres fonctionnels: l'apport de l'école d'Auxerre dans la seconde moitié du IX siècle », in Annales, Économies, Sociétés, Civilisations, janvier-février 1986, t. I, p. 101-126.

ID., «Auxerre, centre Carolingien », in Intellectuels et artistes. .., 1990, p. 199-200.

ID., « D'Auxerre à Canossa en passant par Cluny », in Intellectuels et artistes..., 1990, p. 277-279.

ID., «Haymon », in Intellectuels et artistes..., 1990, p. 36-37.

ID., «Heiric d'Auxerre, Gloses », in Intellectuels et artistes..., 1990, notice ${ }^{\circ} 21$, p. 58.

ID., «Heiric d'Auxerre (?), Florilège érigénien », in Intellectuels et artistes. .., 1990, p. 255-257.

ID., «Heiric d'Auxerre (?), Gloses bibliques », in Intellectuels et artistes..., 1990, notice $\mathrm{n}^{\circ} 22$, p. 59-61.

ID., «Heiric d'Auxerre, Vie de saint Germain », in Intellectuels et artistes. .., 1990, notice $\mathrm{n}^{\circ} 28, \mathrm{p} .70-72$.

ID., «La redécouverte du néoplatonisme », in Intellectuels et artistes..., 1990, p. 254-255.

ID., «Le texte des Miracula sancti Germani et son intérêt pour l'histoire des idées politiques », in Intellectuels et artistes..., 1990, p. 101-104.

ID., « Raoul Glaber, Histoires », in Intellectuels et artistes..., 1990, notice ${ }^{\circ} 170$, p. 279-281.

ID., « Rome et l'antiquité latine», in Intellectuels et artistes..., 1990, p. 258-260. 
ID., "L'œuvre d'Haymon d'Auxerre: état de la question », in L'école carolingienne d'Auxerre..., 1991, p. 157-179.

JaCobsen, Peter Christian, «Die Vita s. Germani Heirics von Auxerre : Untersuchungen zu Prosodie und Metrik», in L'école carolingienne d'Auxerre..., 1991, p. 329-351.

JEAUNEAU, Édouard, «Les écoles de Laon et d'Auxerre au IX ${ }^{\mathrm{e}}$ siècle», in La scuola nell'alto medioevo (Settimane... XIX), Spolète, 1972, p. 495-522 (réimpr. "Les écoles de Laon et d'Auxerre au IX siècle», in Études érigéniennes, Paris, 1987, p. 55-84).

ID., «Heiric d'Auxerre, disciple de Jean Scot», in L'école carolingienne d'Auxerre..., 1991, p. 353-370.

JEUDY, Colette, «Les maîtres: un éclat, une influence», in Intellectuels et artistes..., 1990, p. 32-33.

ID., « Murethach », in Intellectuels et artistes..., 1990, p. 33-34.

ID., « Remi », in Intellectuels et artistes..., 1990, p. 44-47.

ID., «L'œuvre de Remi d'Auxerre: état de la question », in L'école carolingienne d'Auxerre..., 1991, p. 373-397 + p. 459-460.

KUPPER, Jean-Louis, «Saint-Lambert. De l'histoire à la légende», in Revue d'Histoire Ecclésiastique (vol. LXXIX), Louvain, 1984, p. 5-49 (réimpr. Feuillets de la cathédrale de Liège, n 9, 1993) : http://www.ulg.ac.be/trecatlg/VieSL.html.

L'abbaye de Gorze au Xe siècle, Michel PARISSE et Otto Gerhard OEXLE (dir.), Nancy, Presses universitaires de Nancy (Lorraine), 1993, 248 p.

L'école carolingienne d'Auxerre, de Murethach à Remi (830-908), Dominique IOGNA-PRAT, Colette JEUdy et Guy LOBRICHON (dir.), Entretiens d'Auxerre (1989), in Paris, Beauchesne, 1991, 506 p.

«L'exégèse auxerroise», éd. Edmond OrTIGUES et Dominique IOGNA-PRAT, in Intellectuels et artistes..., 1990, p. 62.

Le livre au Moyen Âge, Jean GLENISSON (dir.), Paris, CNRS Édition, 2002, 247 p.

«Le scriptorium de Saint-Germain d'Auxerre?», éd. Claude CouprY et Jean VeZIN, in Intellectuels et artistes..., 1990, p. 27-29.

LECOUTEUX Stéphane, «Une reconstitution hypothétique du cheminement des Annales de Flodoard depuis Reims jusqu'à Fécamp », in Tabularia «Études», $\mathrm{n}^{\circ} 4,2004$, p. 1-38.

LEONARDI, Claudio, "Une école au carrefour de la culture carolingienne », in L'école carolingienne d'Auxerre..., 1991, p. 445-453.

Les gestes des évêques d'Auxerre, éd. et trad. collective, Michel SoT (dir.), Paris, Les-Belles-Lettres (Les classiques de l'Histoire de France au Moyen Âge, 42), 2002, 400 p. 
LE MAHO, Jacques, «La production éditoriale à Jumièges vers le milieu du $\mathrm{X}^{\mathrm{e}}$ siècle », in Tabularia «Études», $\mathrm{n}^{\circ}$ 1, 2001, p. 11-32.

LOBRICHON, Guy, "Gestes des Évêques d'Auxerre», in Intellectuels et artistes..., 1990, notice $\mathrm{n}^{\circ} 176$, p. 286-287.

ID., «Les historiens auxerrois aux XII ${ }^{\mathrm{e}}$ et XIII" siècles », in Intellectuels et artistes..., 1990, p. 284-285.

ID., «Les vies de saint Germain d'Auxerre et les essais hagiographiques de l'école », in Intellectuels et artistes..., 1990, p. 69-70.

ID., «L'atelier auxerrois aux $\mathrm{X}^{\mathrm{e}}$ et $\mathrm{XI}^{\mathrm{e}}$ siècles », in L'école carolingienne d'Auxerre..., 1991, p. 59-69.

Lusse, Jackie, Laon et le Laonnois $d u V^{e}$ au $X^{e}$ siècle, Nancy, Presse Universitaire de Nancy, 1992, 424 p.

MANiTIUS, Max, Geschichte der lateinischen Literatur des Mittelalters, Müchen, Beck, 1974 (rééd. 1911), XIII-766 p.

Manuscrits datés des Bibliothèques de France, dir. Denis Muzerelle, Paris, CNRS, 2000, t. I (Cambrai), XXVI-330 p.

MOLINIER, Auguste, Les sources de l'Histoire de France, Paris, Picard, 1901, t. I, VIII +288 p.

NeLSON, Janet L., "Charles le Chauve et les utilisations du savoir », in L'école carolingienne d'Auxerre..., 1991, p. 37-54.

ORTigues, Edmond, "Haymon d'Auxerre, théoricien des trois ordres », in L'école carolingienne d'Auxerre..., 1991, p. 181-227.

PICARD, Jean-Charles, «Les églises d'Auxerre à la fin du VIe siècle», in Intellectuels et artistes..., 1990, p. 6-8.

ID., «Une lecture archéologique du texte d'Heiric», in Intellectuels et artistes..., 1990, p. 105-108.

Pithou, François et Pierre, «Ordo Cameracensis et Atrebatensis ecclesiae », in Codex Canonum vetus ecclesiae Romanae, Paris, Typographia regia, 1687, p. 367-369.

QUADRI, Riccardo, « Heiric», in Intellectuels et artistes..., 1990, p. 37-40.

ID., "Heiric d'Auxerre, Calendrier », in Intellectuels et artistes..., 1990, notice $\mathrm{n}^{\circ} 14$, p. 41-42.

ID., «Heiric d'Auxerre, Collectanea », in Intellectuels et artistes..., 1990, notice $\mathrm{n}^{\circ} 167$, p. 269.

RICHÉ, Pierre, Écoles et enseignements dans le Haut Moyen Âge (dans l'Occident chrétien, de la fin du $V^{e}$ au milieu du XI e siècle), Paris, Aubier-Montaigne, 1979, 462 p. 
SAINT-DENIS, Alain, Apogée d'une cité: Laon et le Laonnois aux XII et XIII siècles, Nancy, Presses universitaires de Nancy (Collection Histoire et archéologie médiévales), 1994, 652 p.

SAPIN, Christian, "Carte des relations entre Auxerre et la Francie orientale », in Intellectuels et artistes..., 1990, p. 229-232.

ID., «Ferrières-en-Gâtinais (Loiret). Plan de l'église abbatiale », in Intellectuels et artistes..., 1990, notice $\mathrm{n}^{\circ} 130$, p. 208-209.

SASSIER, Yves, «Les Carolingiens et Auxerre», in L'école carolingienne d'Auxerre..., 1991, p. 21-36.

SHOPKOw, Leah, «The Carolingian world of Dudo of Saint-Quentin », in Journal of Medieval History, $\mathrm{n}^{\circ} 15,1989$, p. 19-37.

Sot, Michel, Un historien et son Église au Xe siècle: Flodoard de Reims, Paris, Fayard, 1993, 832 p.

TilliETTE, Jean-Yves, «Métrique carolingienne et métrique auxerroise: quelques réflexions sur la Vita sancti Germani d'Heiric d'Auxerre», in L'école carolingienne d'Auxerre..., 1991, p. 314-327.

VEZIN, Jean, «Le scriptorium d'Auxerre », in L'école carolingienne d'Auxerre..., 1991, p. 57-58. 


\section{Annexes}

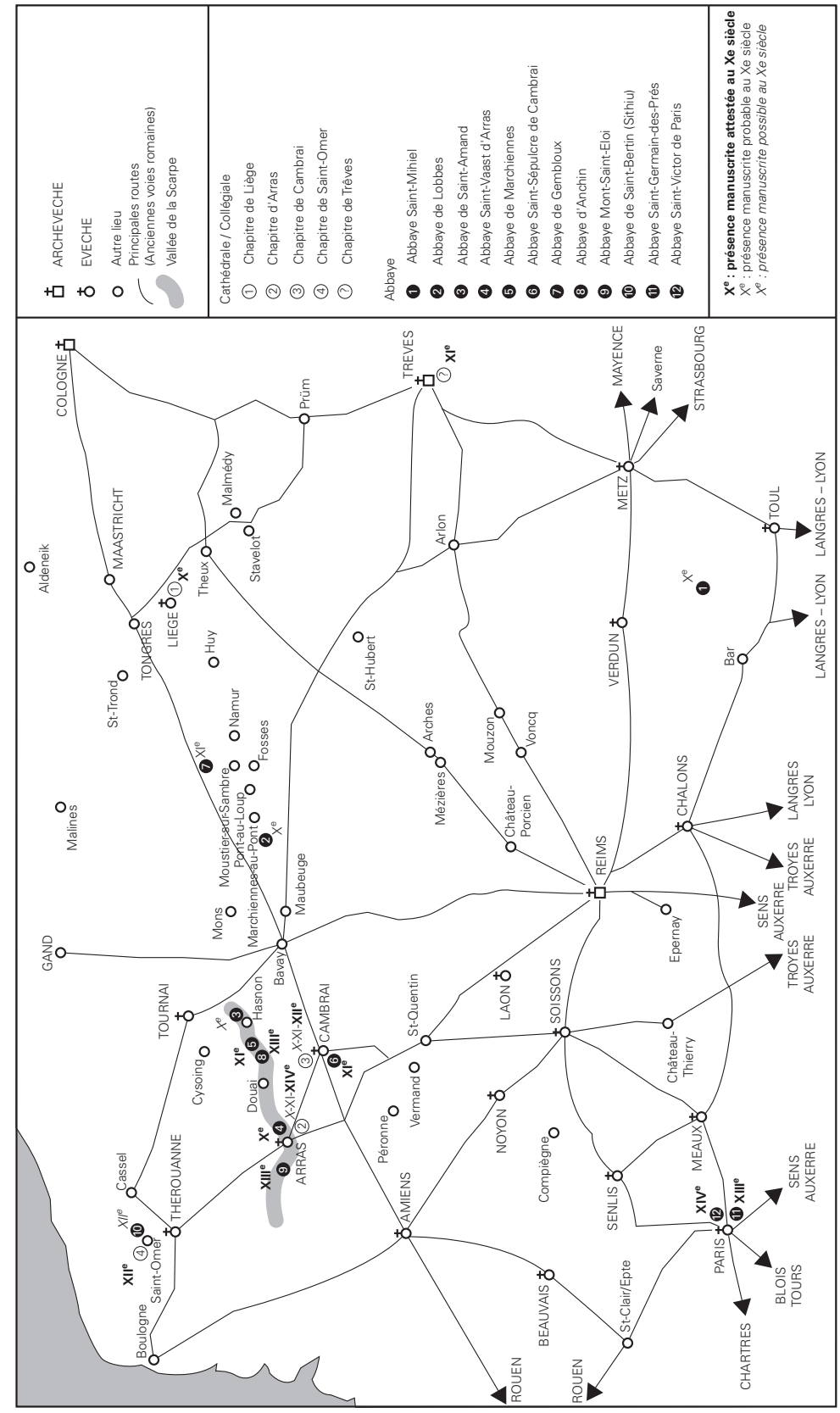

Carte 1 : Répartition géographique occidentale des manuscrits renfermant la Vita sancti Lamberti de l'évêque Étienne de Liège 


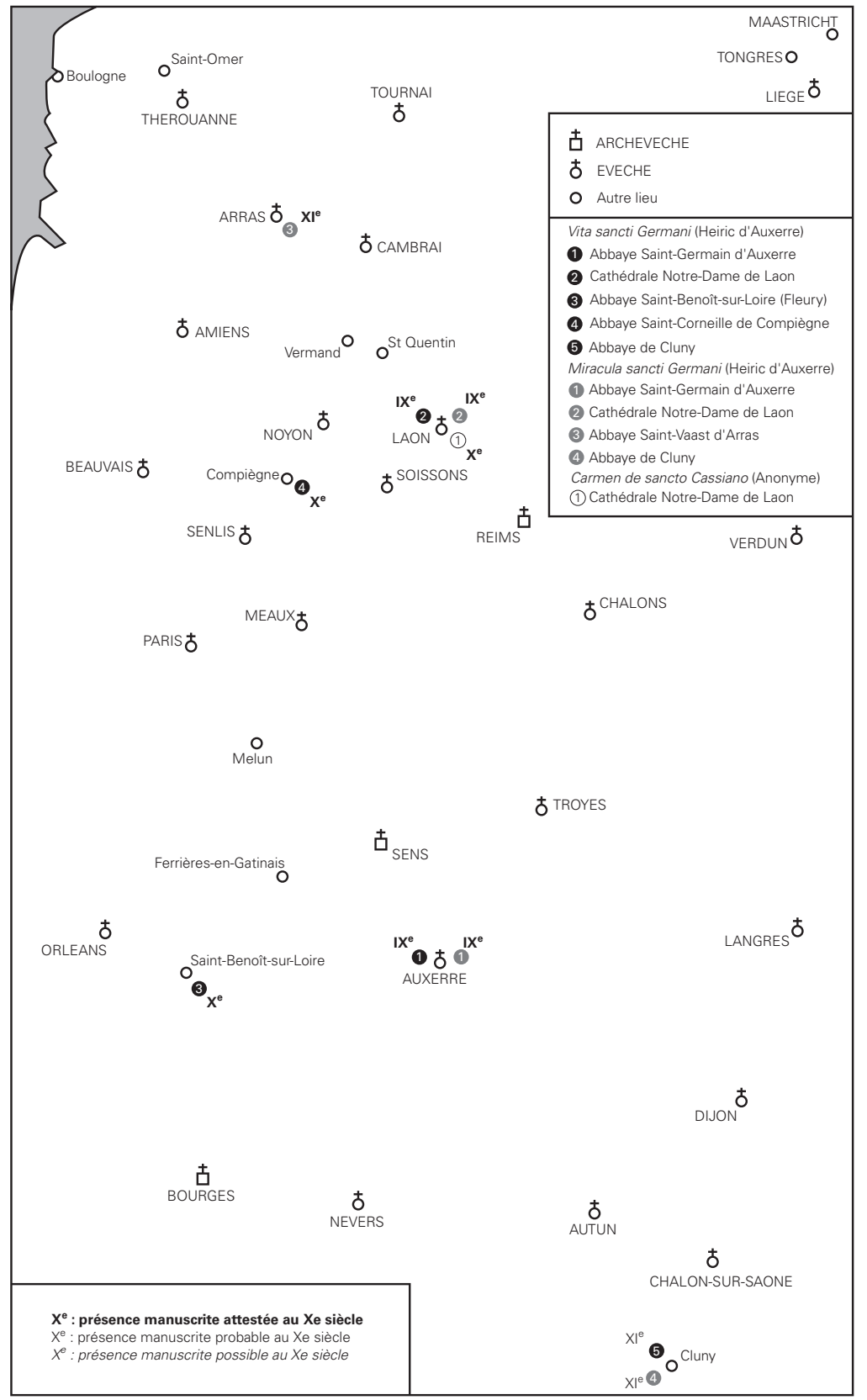

Carte 2 : répartition des manuscrits renfermant la Vita sancti Germani d'Heiric d'Auxerre et le Carmen de sancto Cassiano à l'époque de Dudon de Saint-Quentin. 